\title{
Extracting Microfacet-based BRDF Parameters from Arbitrary Materials with Power Iterations
}

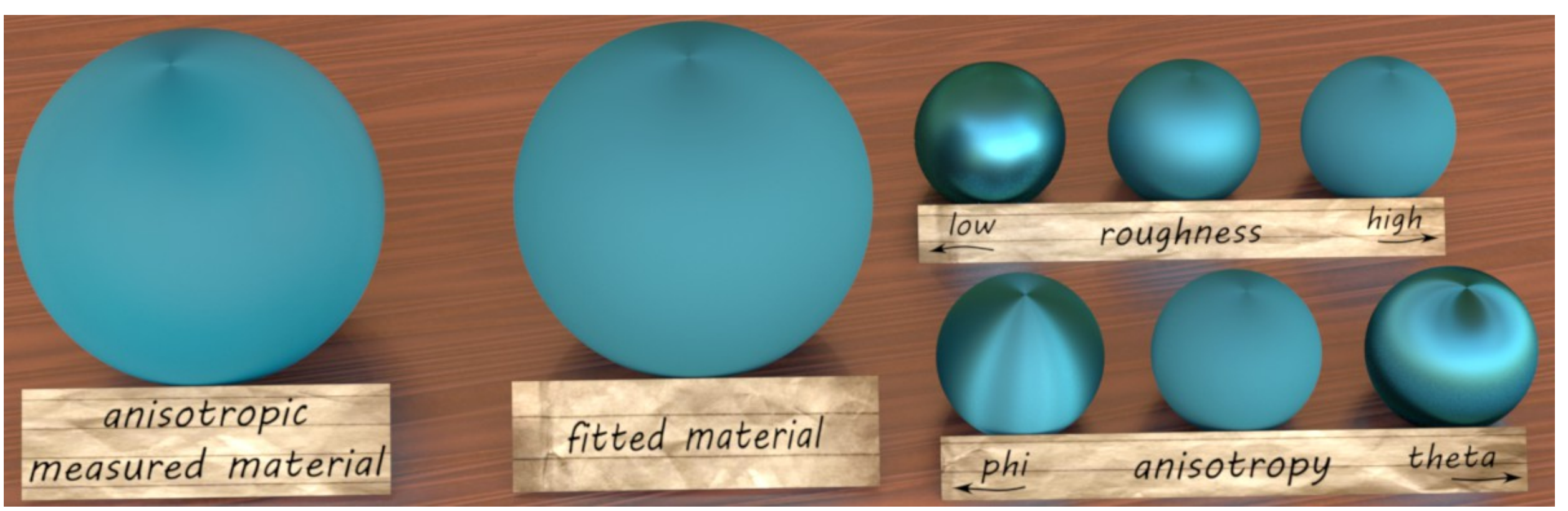

Jonathan Dupuy ${ }^{1,2}$ Eric Heitz ${ }^{3}$ Jean-Claude lehl ${ }^{1} \quad$ Pierre Poulin ${ }^{2}$ Victor Ostromoukhov ${ }^{1}$ 


\section{Talk's Topic: BRDFs}

$$
L_{o}=\int_{\Omega_{+}} L_{i} \cdot f_{r} \cdot \cos \theta_{i} \cdot d \omega_{i}
$$

And more particularly:

Physically based microfacet BRDFs
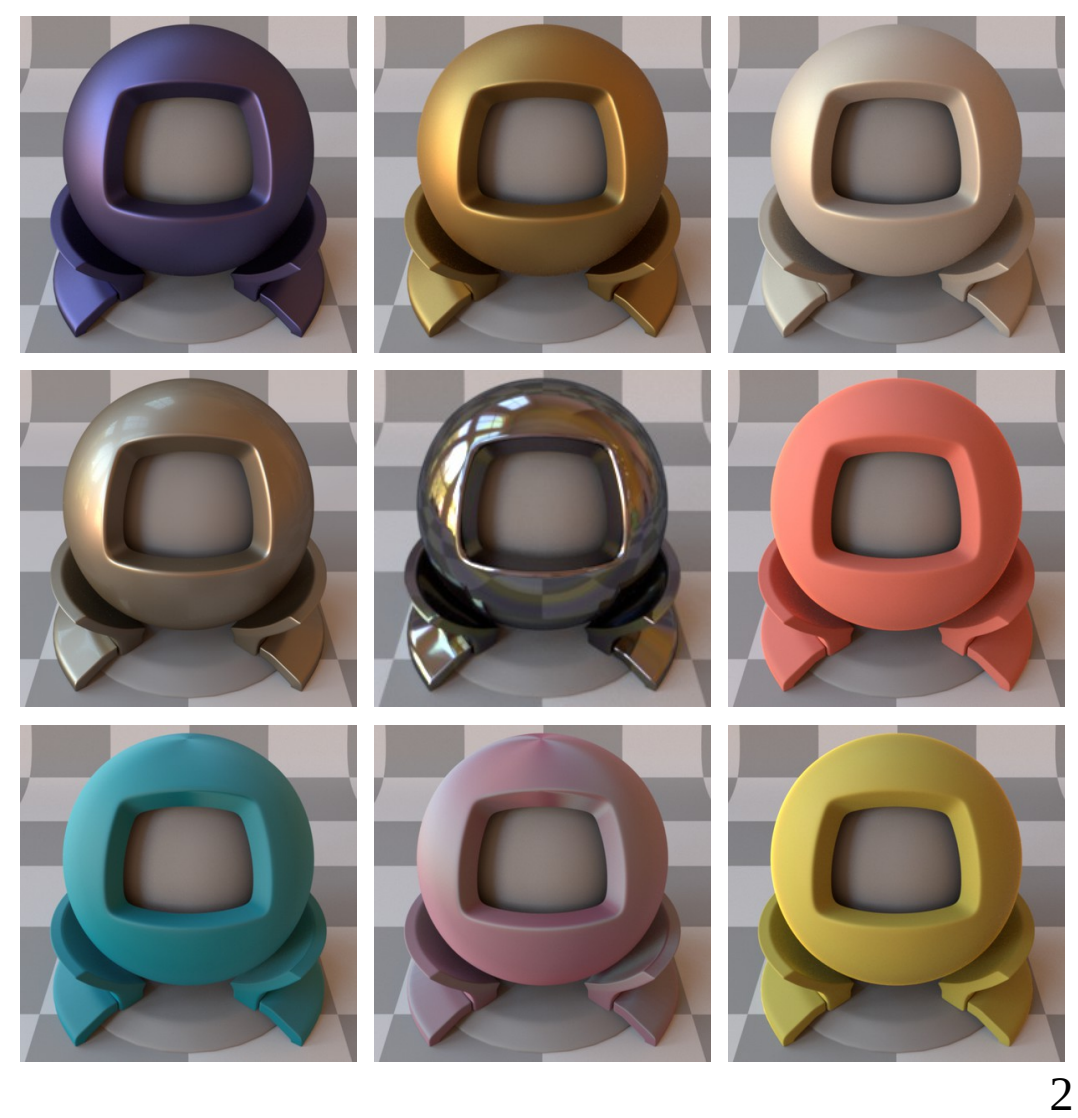


\section{Context}

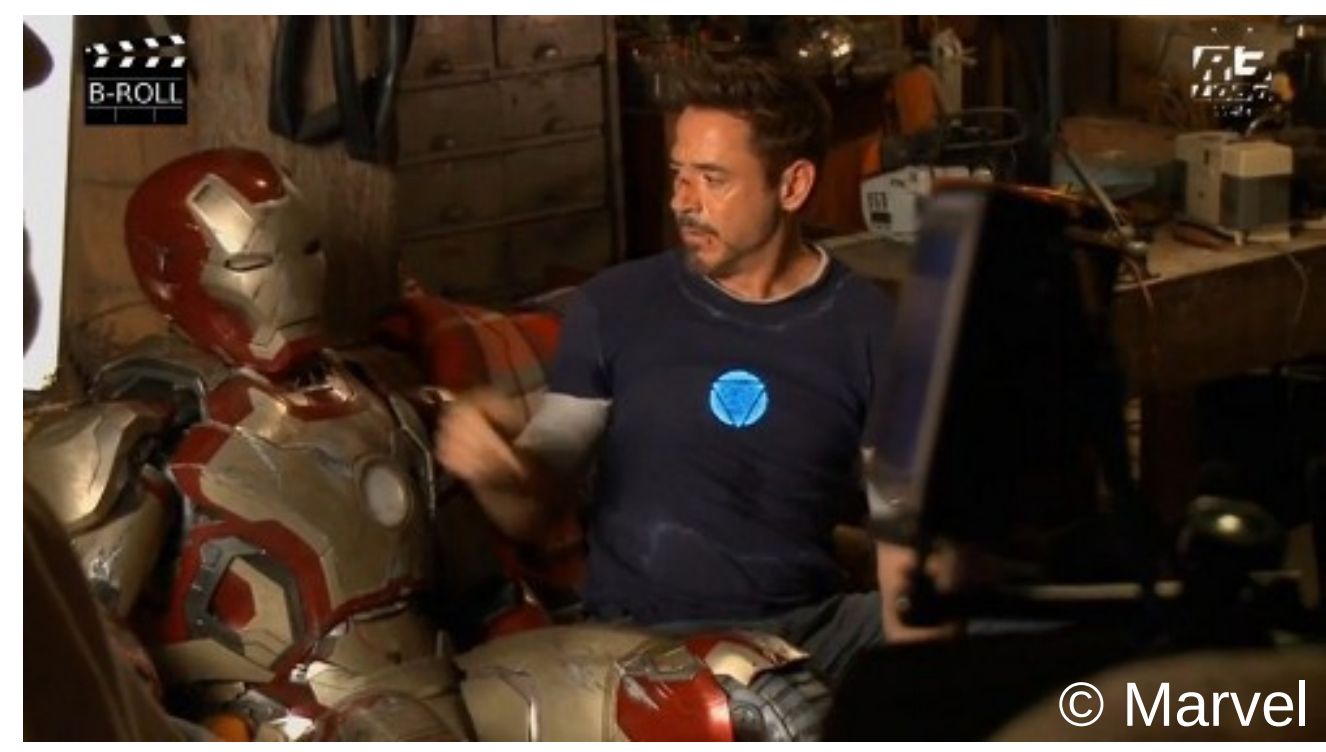

Real

(real materials)

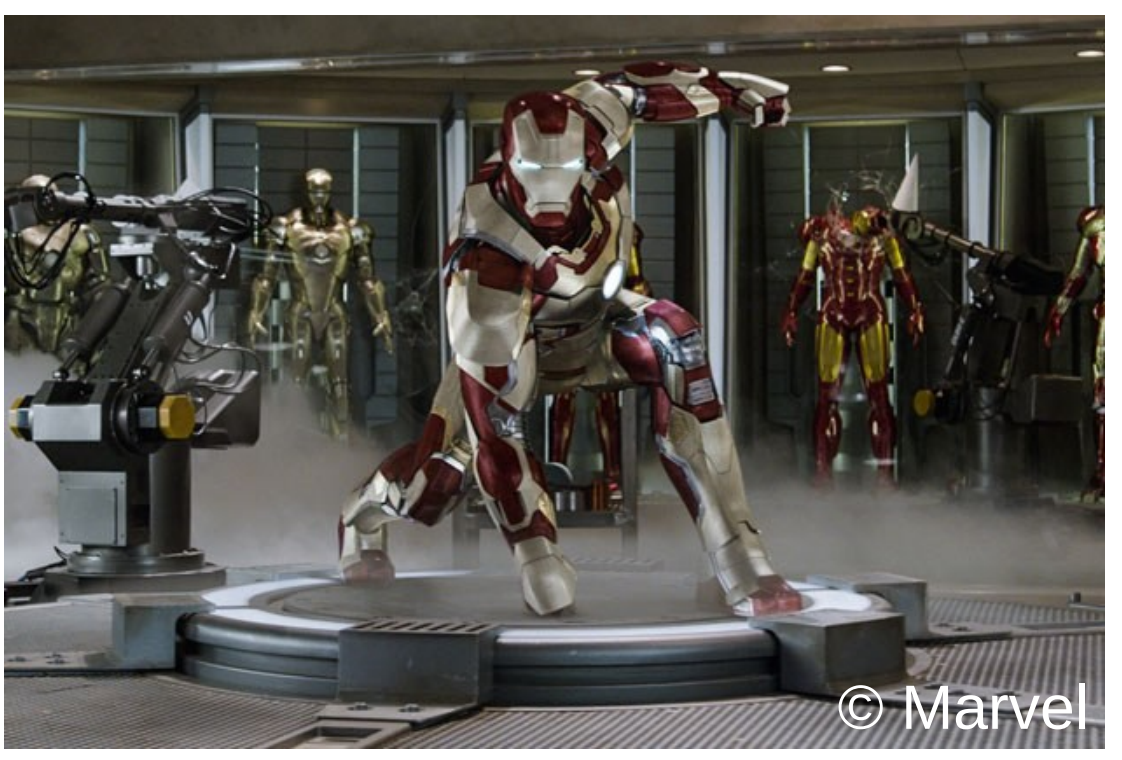

Digital

(microfacet BRDFs) 


\section{Microfacet Theory}

$$
f_{r}=\frac{F \cdot D \cdot G}{4 \cdot \cos \theta_{i} \cdot \cos \theta_{o}}
$$

Intuition

- Materials = microsurfaces

- Artists manipulate microsurface properties (NDF, roughness, etc.)
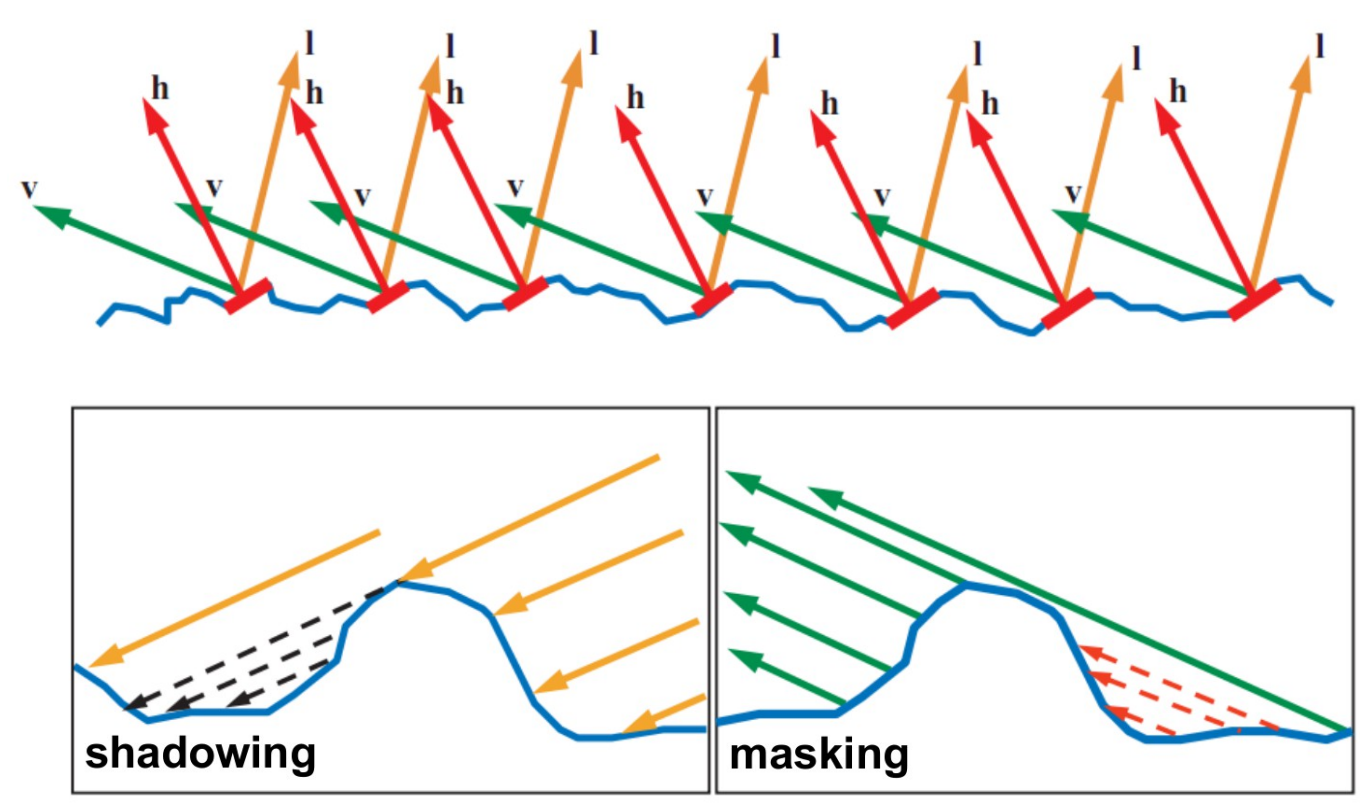


\section{Problem}

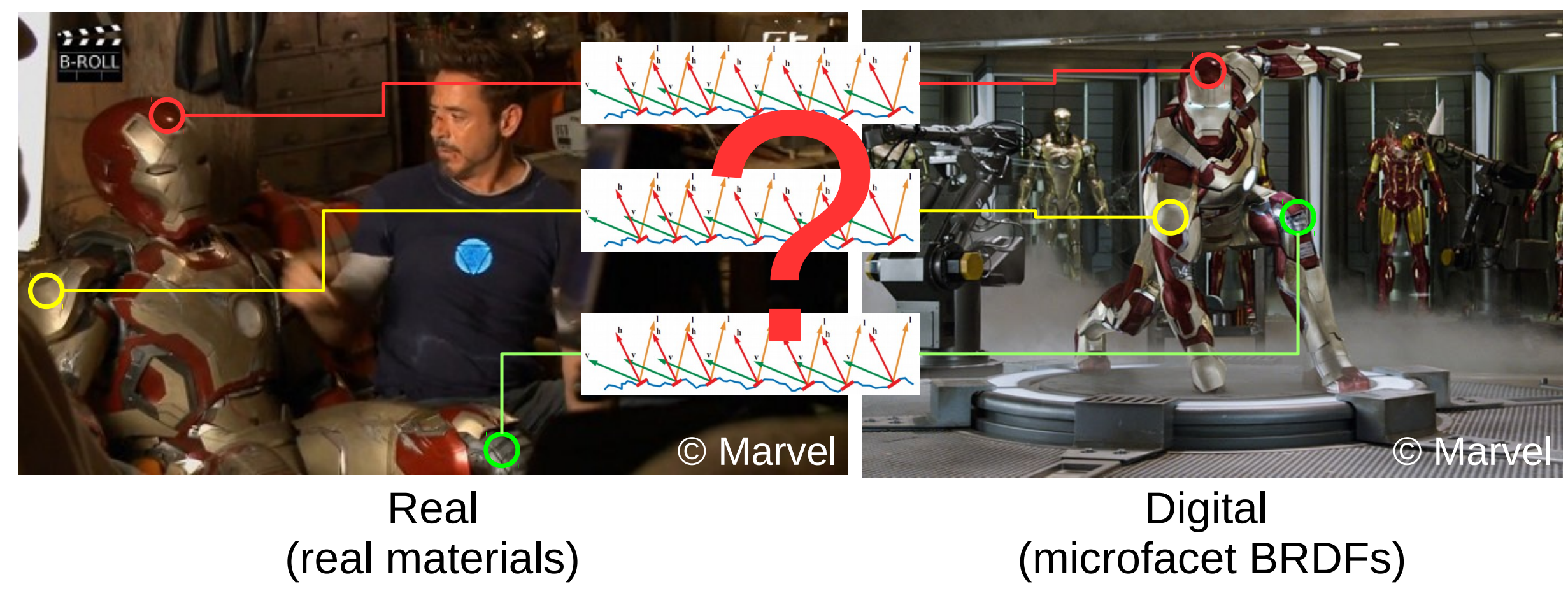

II $\rightarrow$ Can we retrieve the microsurface of real materials ? 


\section{Microfacet BRDF Fitting}

Approach

[NDM05, LKYU12, BSH12, WZT*08]

- Fitted microsurface

- Minimize fitting metric

Current limitations

- Robustness / Speed

- Arbitrary metrics

- Reproducibility

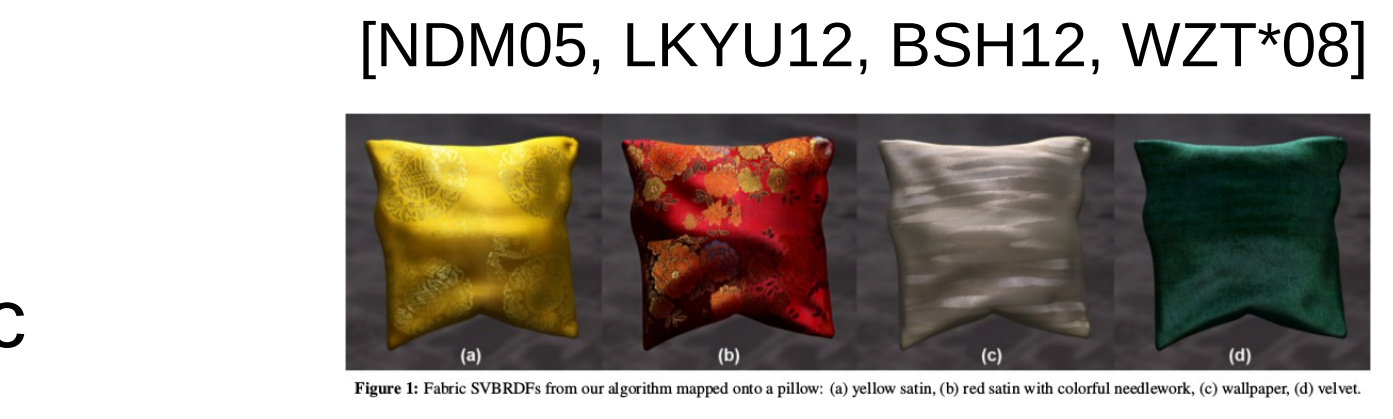

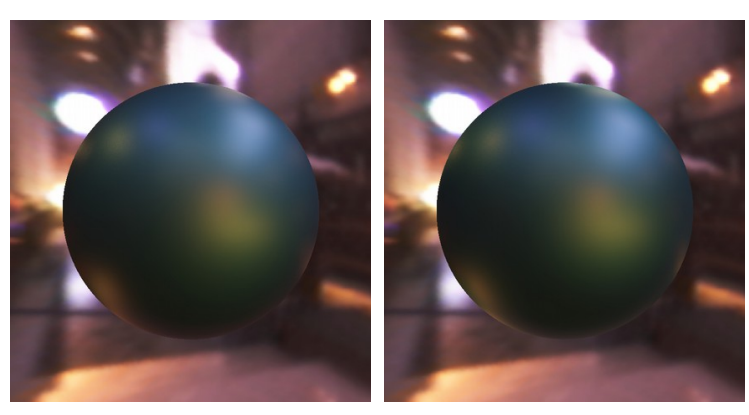

Acquired

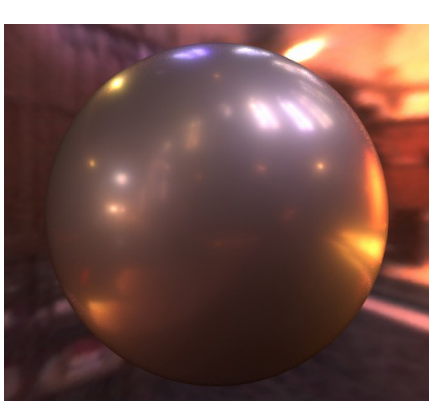

Acquired

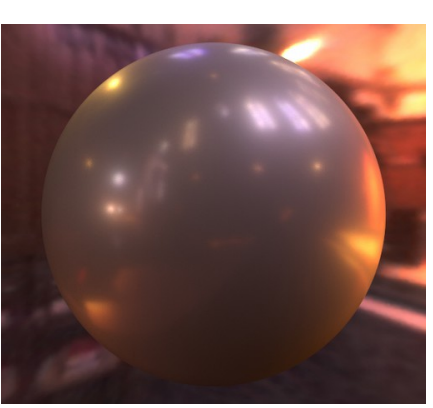

[LKYU12] 


\section{Microfacet BRDF Fitting}

\section{Approach}

- Fitted microsurface

- Minimize fitting metric

Current limitations

- Robustness / Speed

- Arbitrary metrics

- Reproducibility

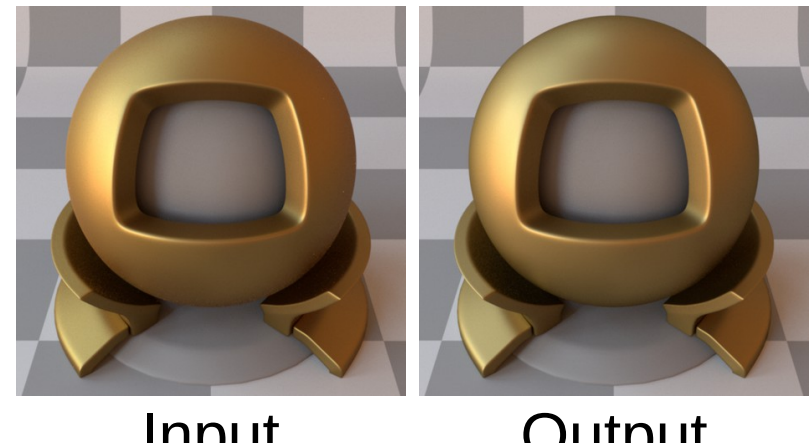

$$
\text { Input }
$$

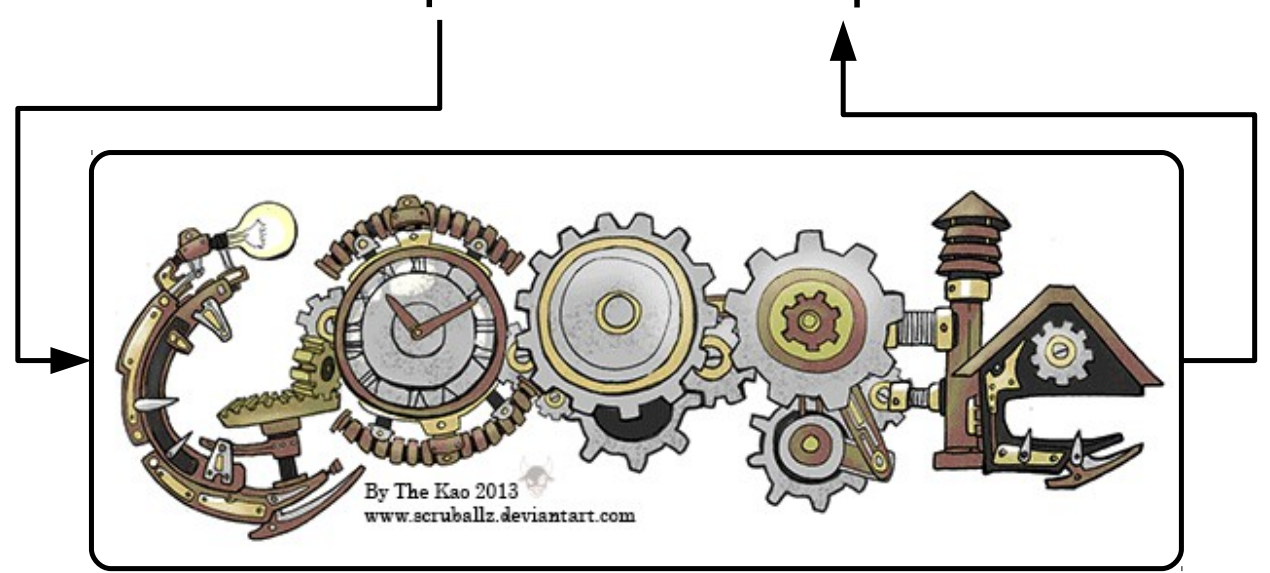

Fitting algorithm 


\section{Microfacet BRDF Fitting}

\section{Approach}

- Fitted microsurface

- Minimize fitting metric

Current limitations

- Robustness / Speed

- Arbitrary metrics

- Reproducibility
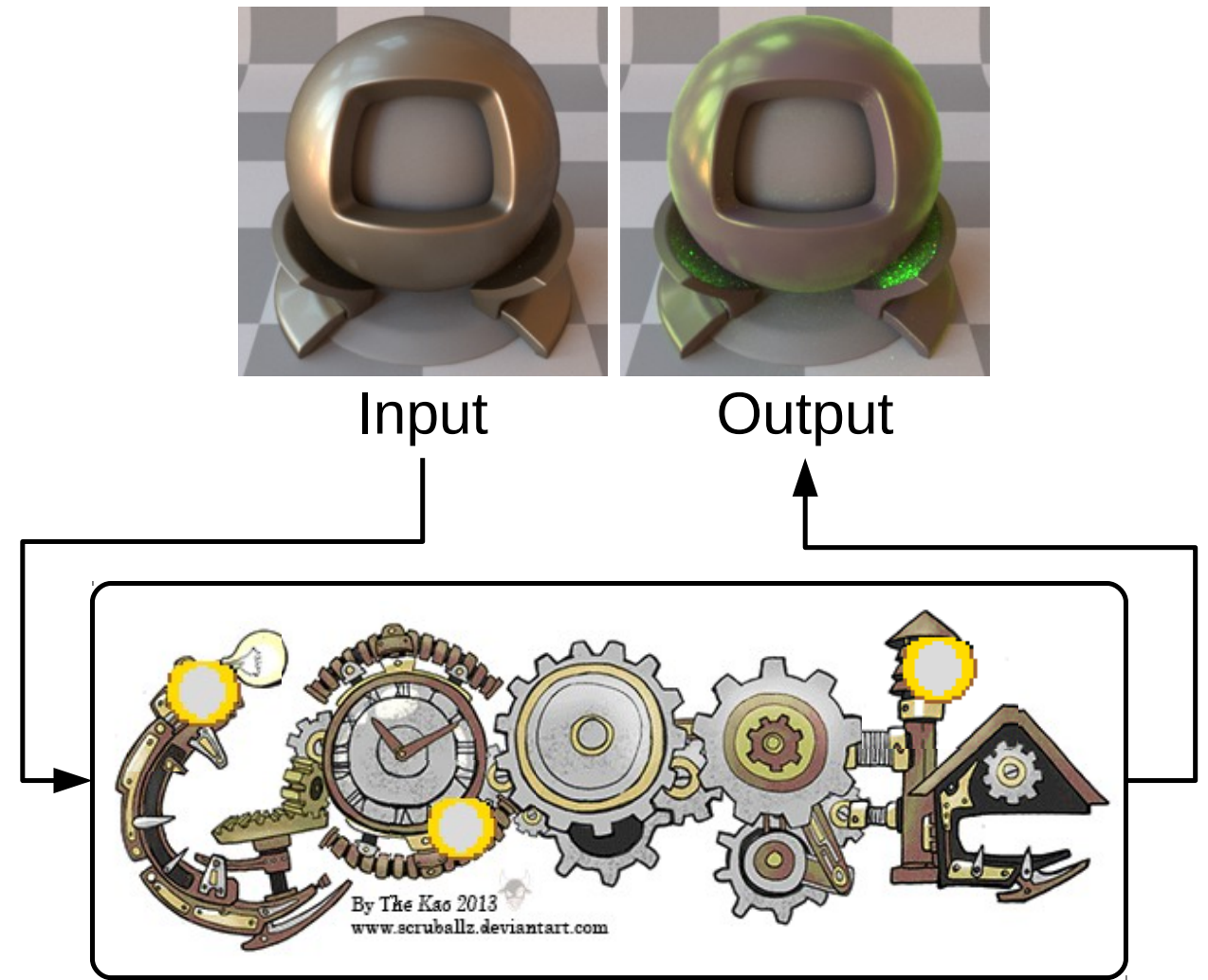

Fitting algorithm 


\section{Microfacet BRDF Fitting}

Approach

- Fitted microsurface

L1-norm ?

Lø-norm ?

- Minimize fitting metric

Current limitations

Log space?

- Robustness / Speed

- Arbitrary metrics

- Reproducibility

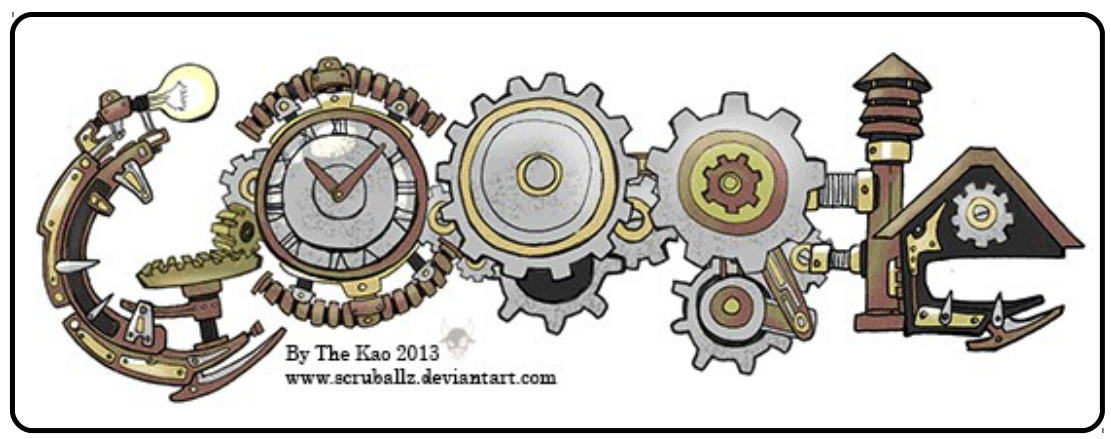

Fitting algorithm 


\section{Microfacet BRDF Fitting}

Approach

- Fitted microsurface

- Minimize fitting metric

Current limitations

- Robustness / Speed

- Arbitrary metrics

- Reproducibility
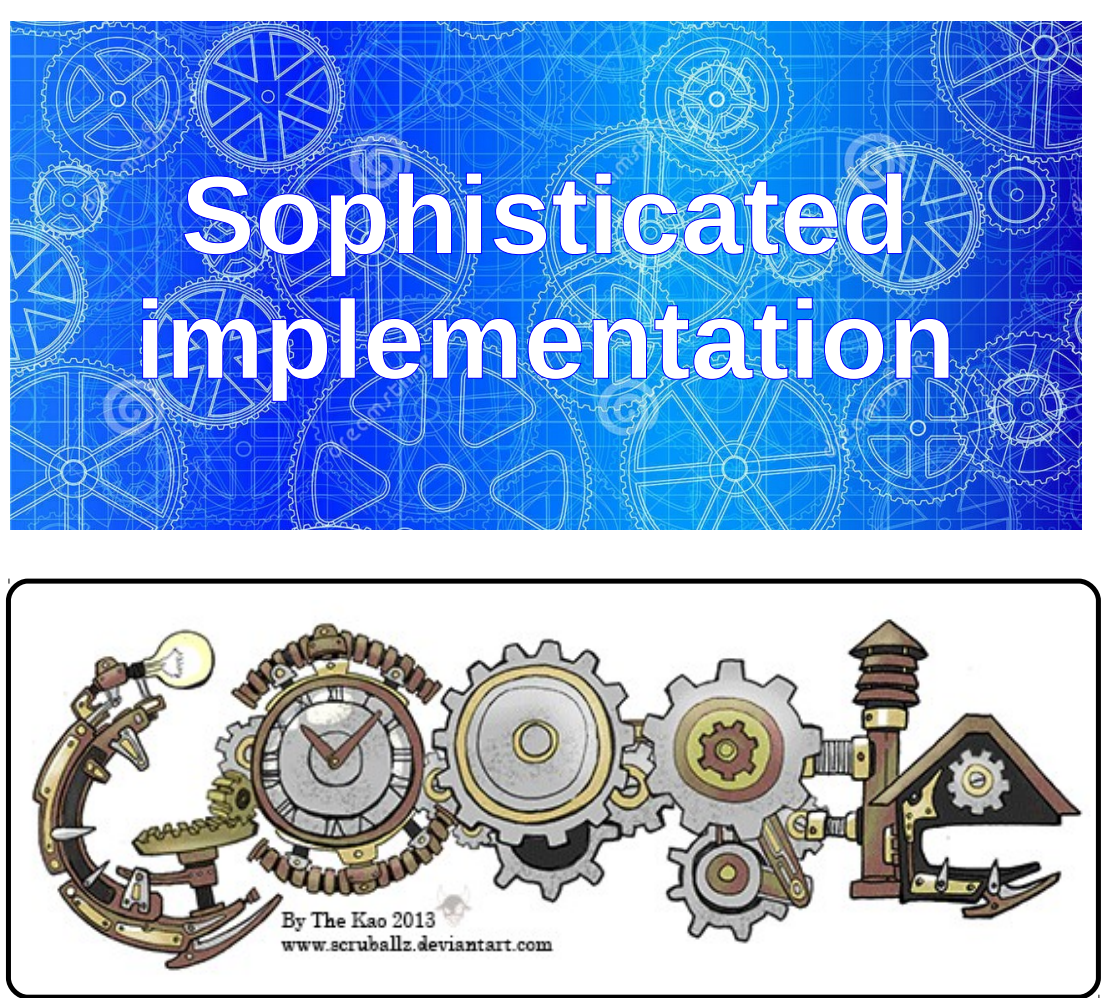

Fitting algorithm 


\section{Contribution}
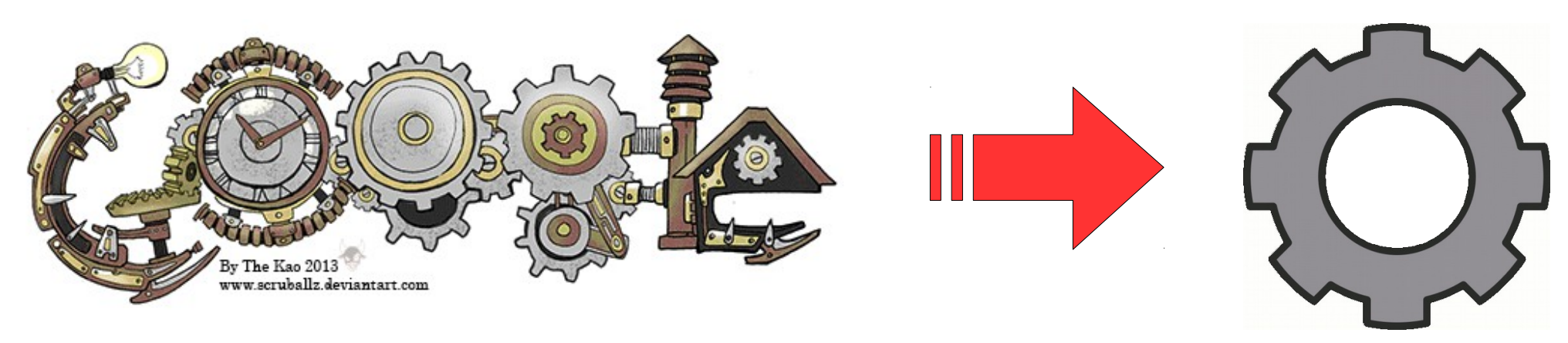


\section{Contribution}

Overview

- Robust tabulation

- Robust parametric fits

Properties

- Robustness

- Simplicity

- Speed

- Reproducibility

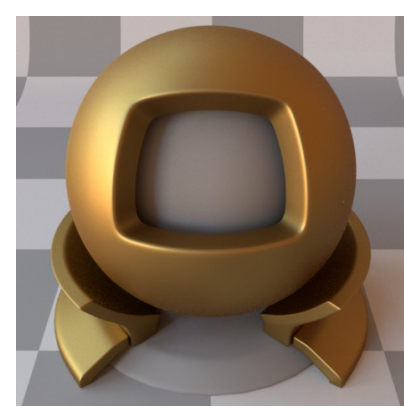

input

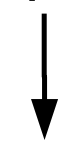

NDF

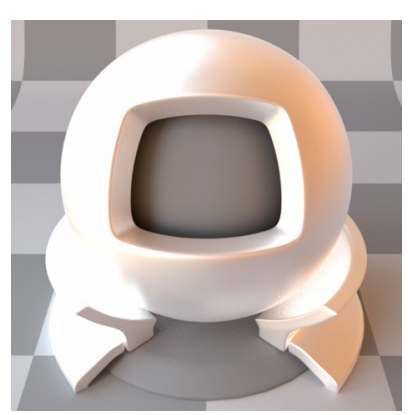

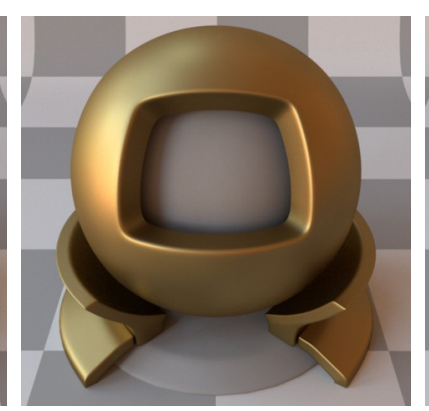

Tabulated
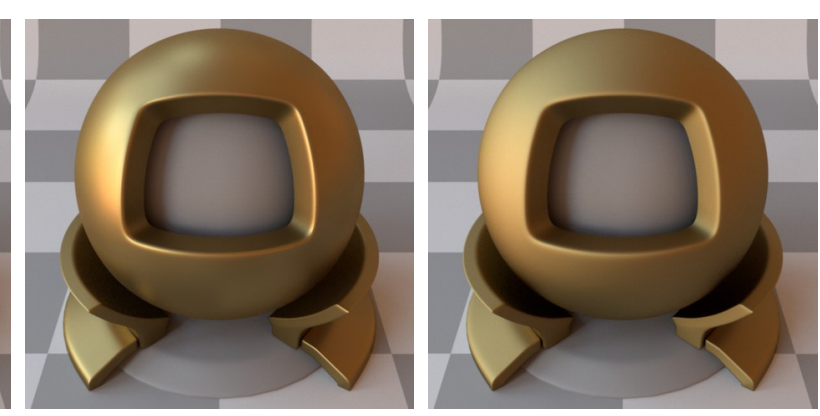

Beckmann

4 


\section{Microfacet Theory}

$$
f_{r}=\frac{F \cdot D \cdot G}{4 \cdot \cos \theta_{i} \cdot \cos \theta_{o}}
$$

Intuition

- Materials = microsurfaces

- Artists manipulate microsurface properties (NDF, roughness, etc.)
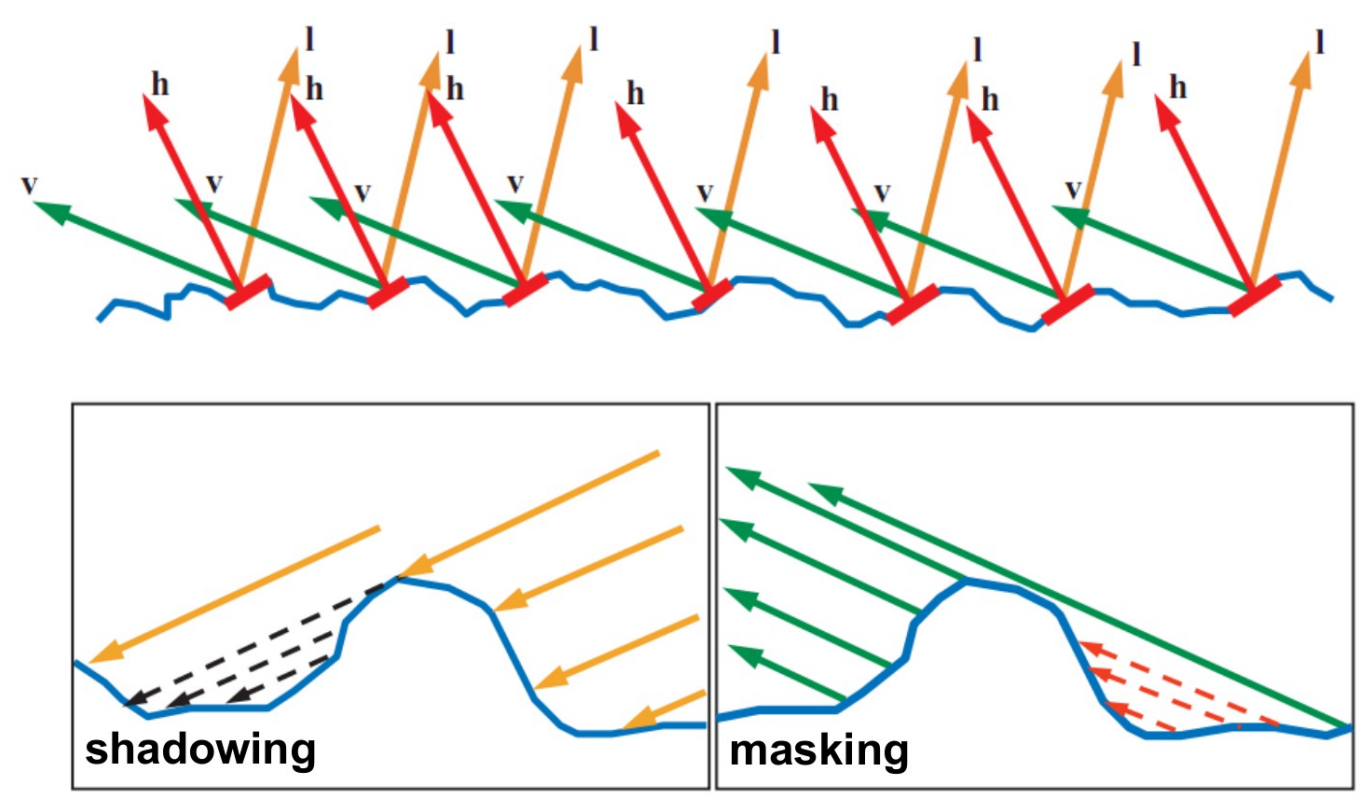


\section{Backscattering Equation}

Mathematical (previous work)

$$
f_{r}=\frac{F_{0} \cdot D \cdot G}{4 \cdot \cos ^{2} \theta_{o}}
$$

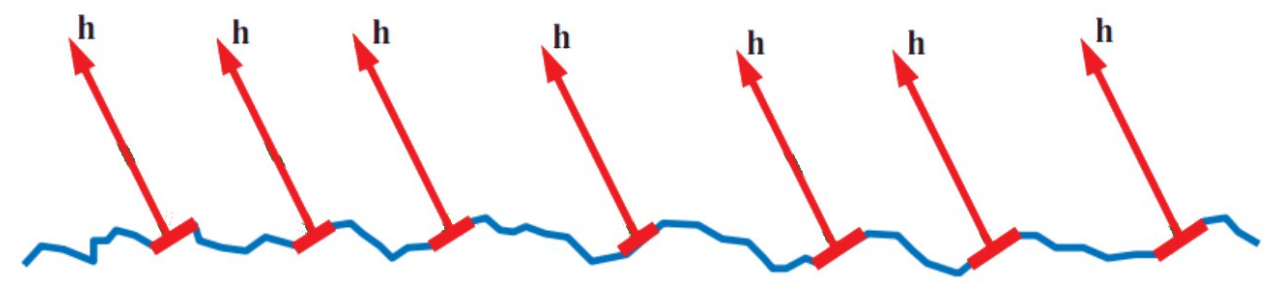

Physical (our work)

$$
f_{r}=\frac{F_{0} \cdot D \cdot G_{1}}{4 \cdot \cos ^{2} \theta_{o}}
$$

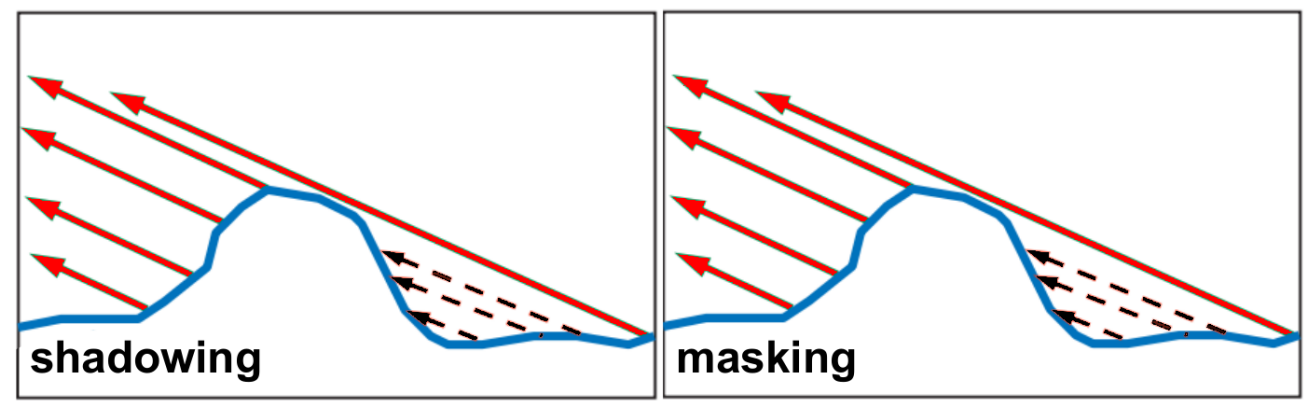




\section{NDF Extraction}

Physical equation

$$
f_{r}=\frac{F_{0} \cdot D \cdot G_{1}}{4 \cdot \cos ^{2} \theta_{o}}
$$

Inverted equation

$$
F_{0} D(\mathbf{o})=\int_{\Omega_{+}} K(\mathbf{o}, \mathbf{h}) D(\mathbf{h}) d \omega_{h}
$$

(Fredholm equation of the second kind)

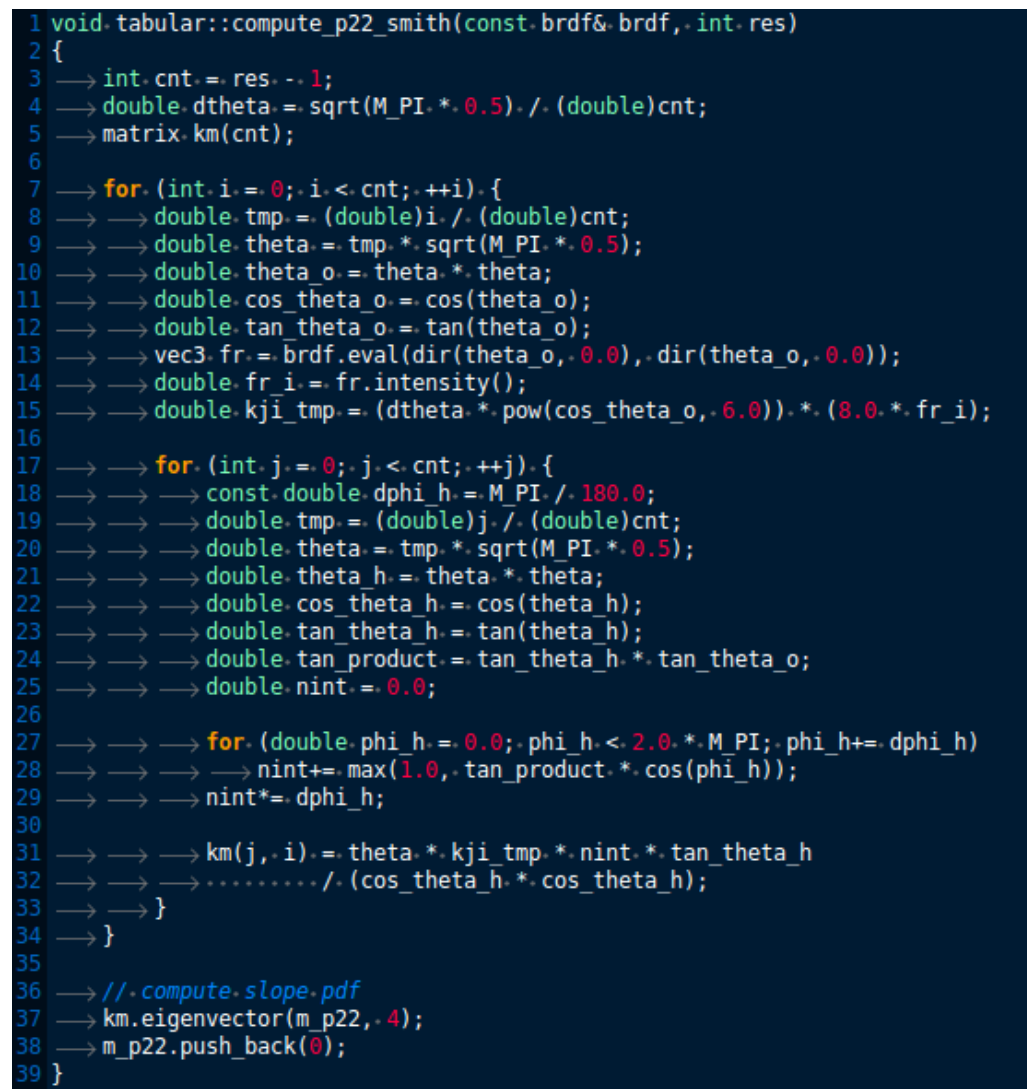




\section{Ideal Mirrors}

$$
f_{r, i d}=\frac{D \cdot G}{4 \cdot \cos \theta_{i} \cdot \cos \theta_{o}}
$$

Special microfacet BRDF

- Fresnel term is 1

- Independent of wavelength

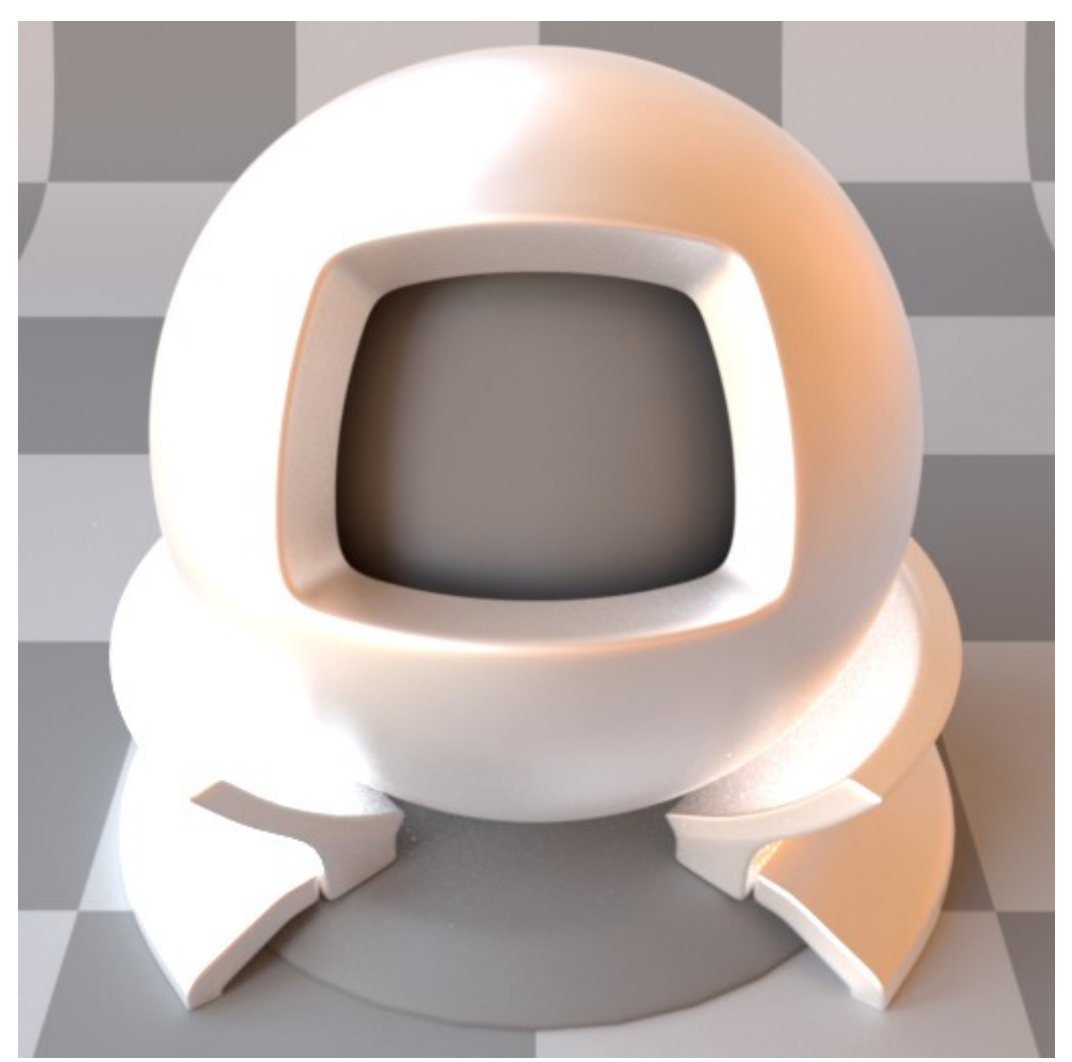




\section{Fresnel Extraction}

$F\left(\theta_{d}\right)=\mathbb{E}\left[\frac{f_{r}}{f_{r, i d}} \mid \mathbf{i h}=\cos \theta_{d}\right]$

We compute an average response

- Fully automatic

- Simple implementation

- Fast evaluation

- Works well in practice

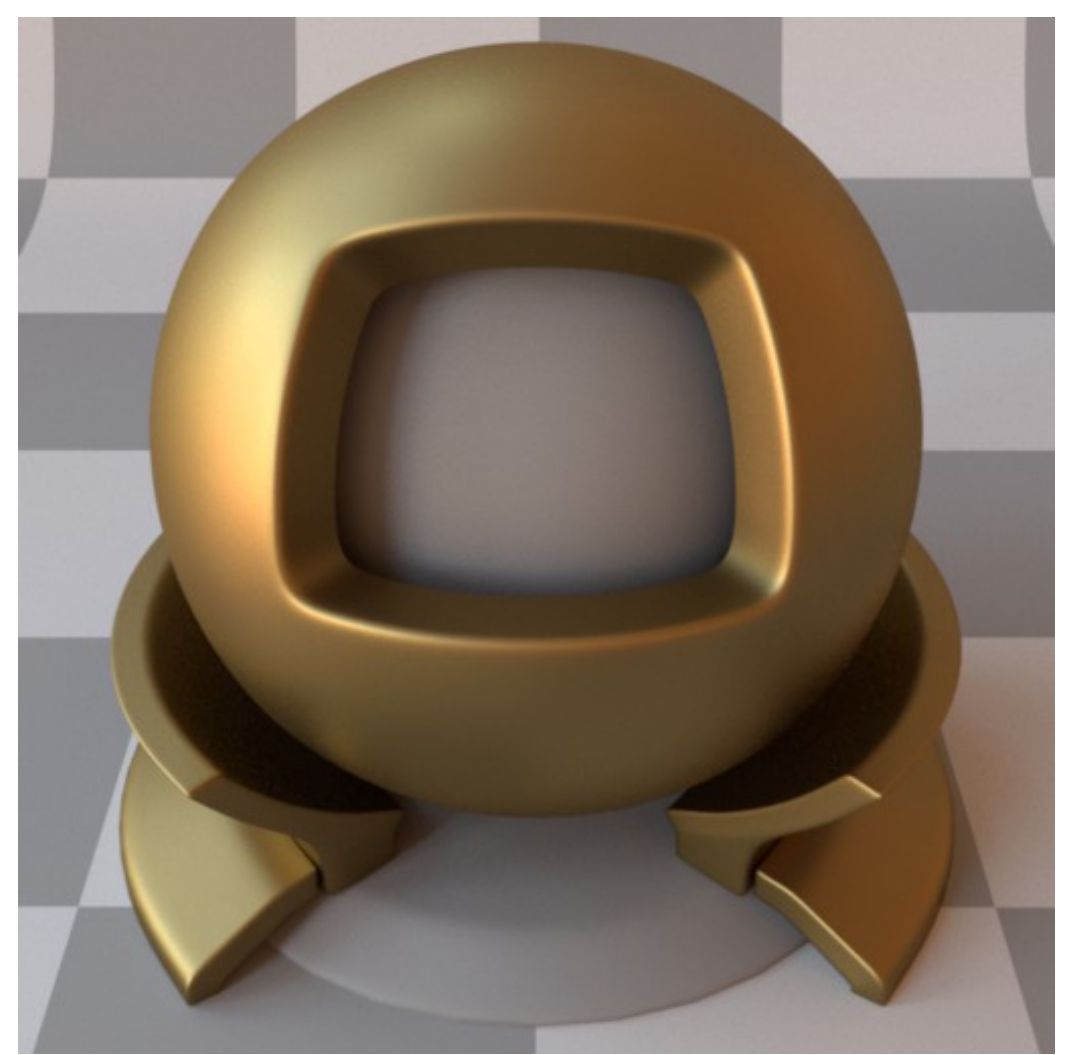




\section{MERL gold-metallic-paint}

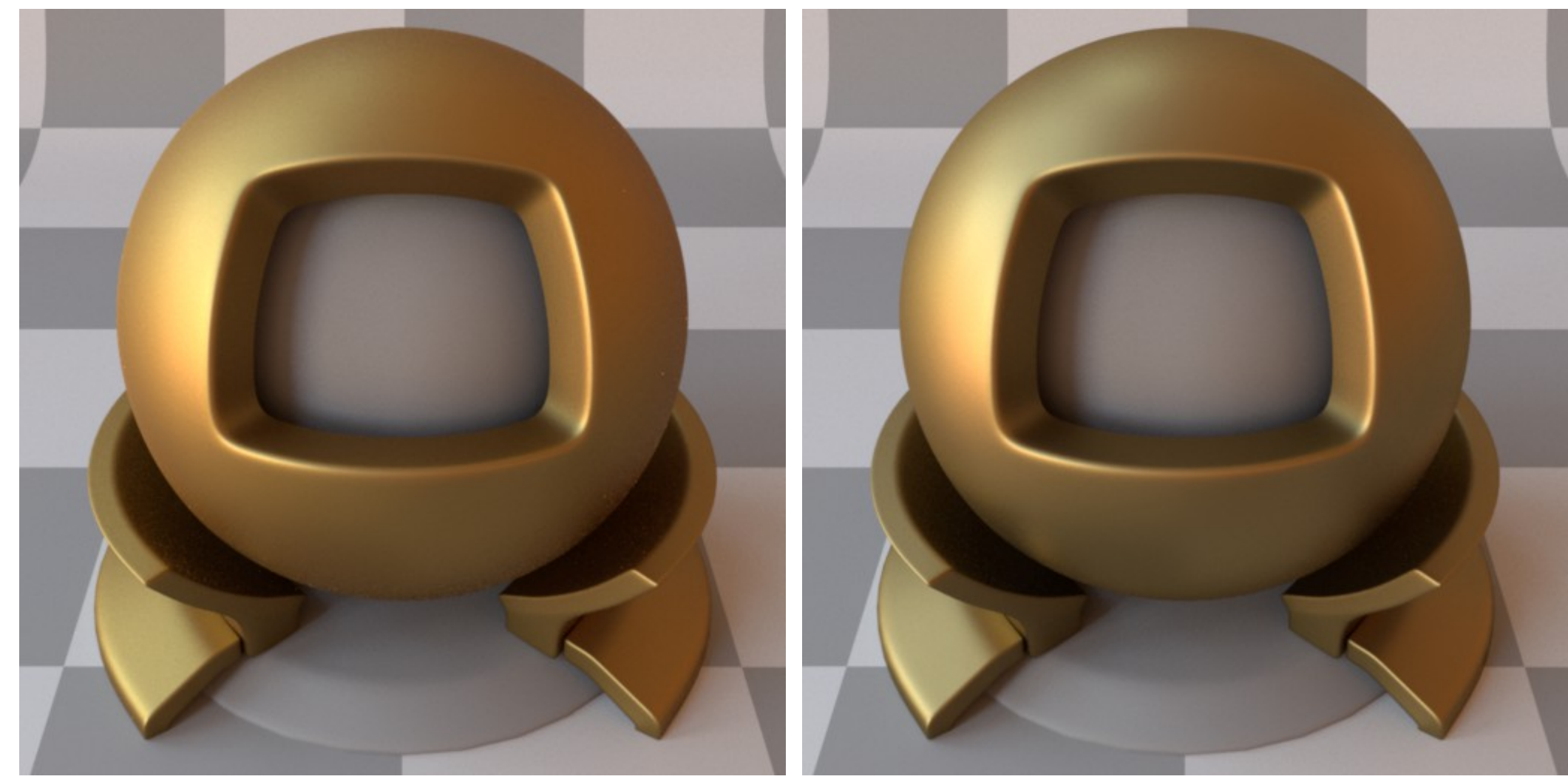




\section{UTIA m064-fabric099}

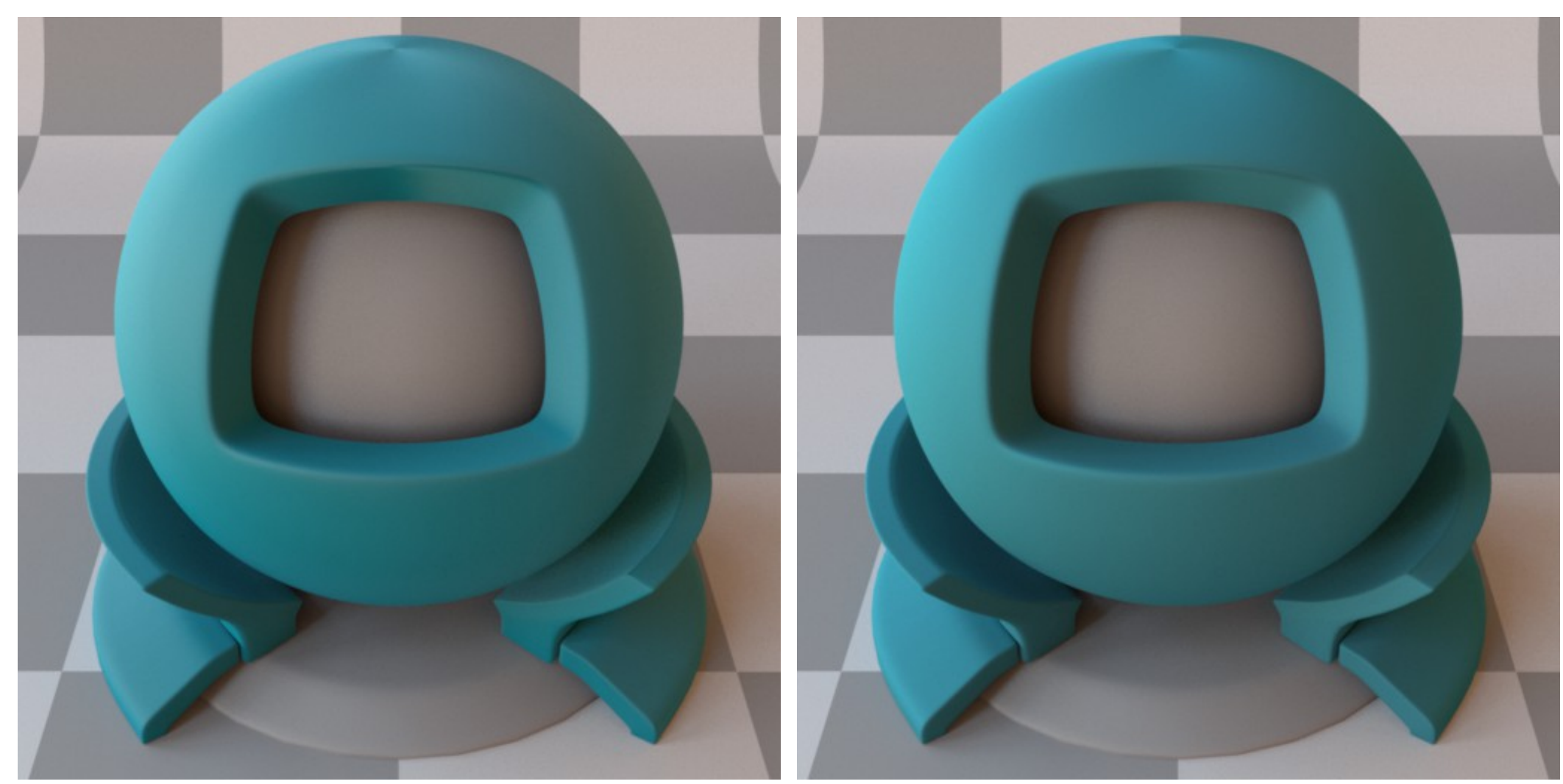




\section{Parametric Fits}

Beckmann roughness

$$
2 \alpha_{G}^{2}=\int_{\mathbb{R}^{2}} \tilde{x}_{h}^{2} P(\tilde{\mathbf{h}}) d \tilde{\mathbf{h}}
$$

GGX roughness

$$
\alpha_{X}=\int_{\mathbb{R}^{2}}\left|\tilde{x}_{h}\right| P(\tilde{\mathbf{h}}) d \tilde{\mathbf{h}}
$$

Our observation: GGX > Beckmann

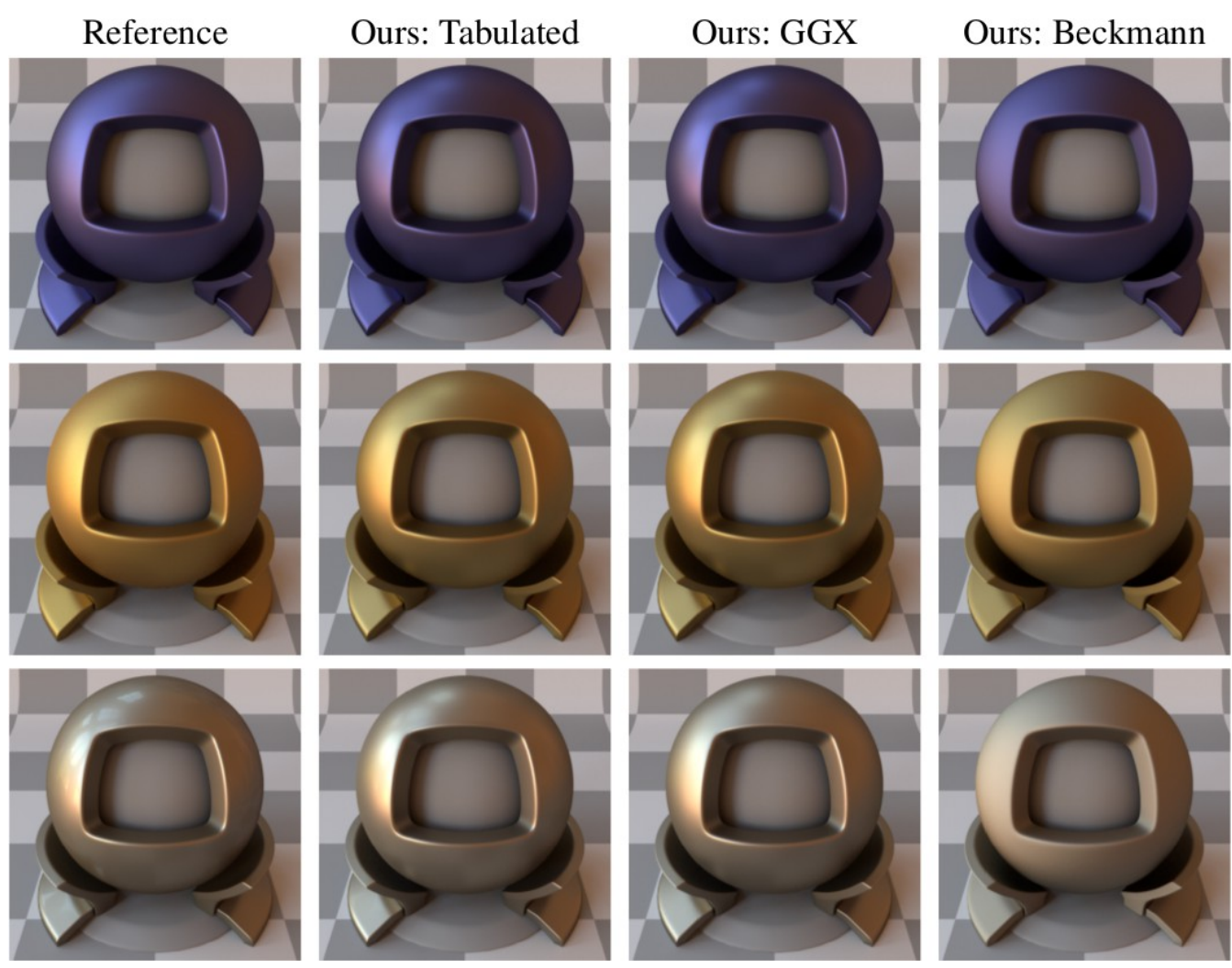




\section{Validation}

29 gold-metallic-paint

Renderings
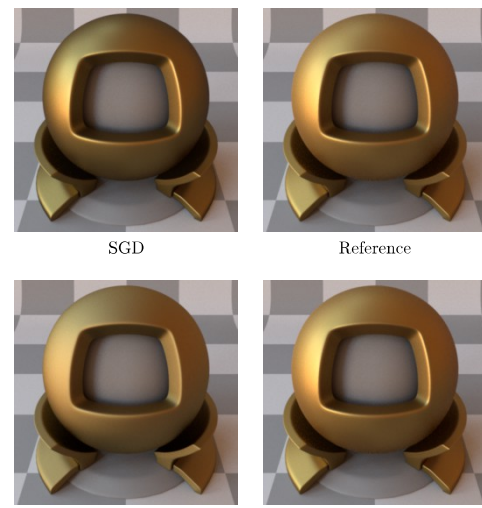

Ours: Gaussian

CIE dE00
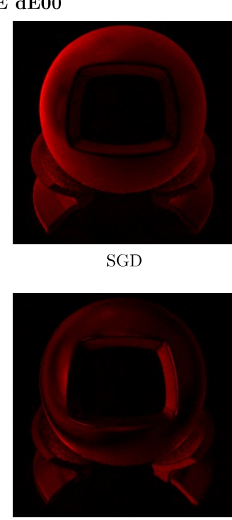

Ours: Gaussian

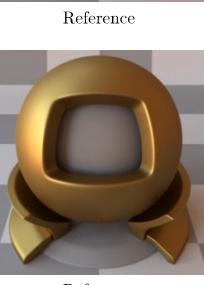

Reference
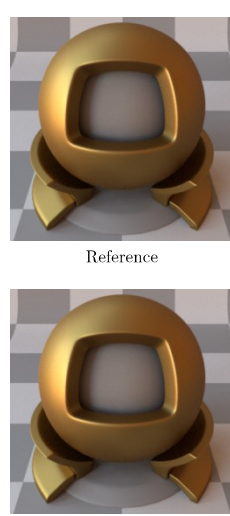
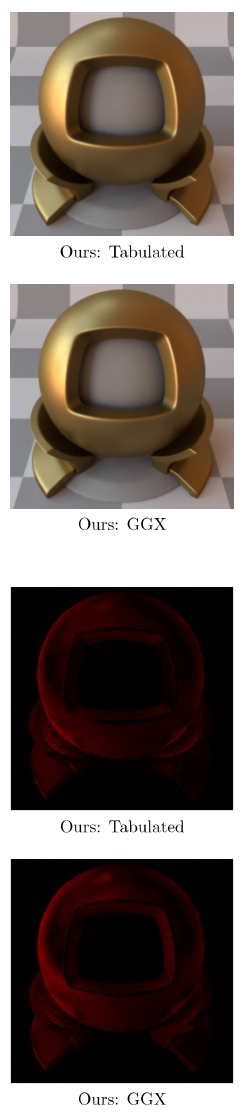

BRDF Lobes in the Incidence Plane (Cubic Root Applied)
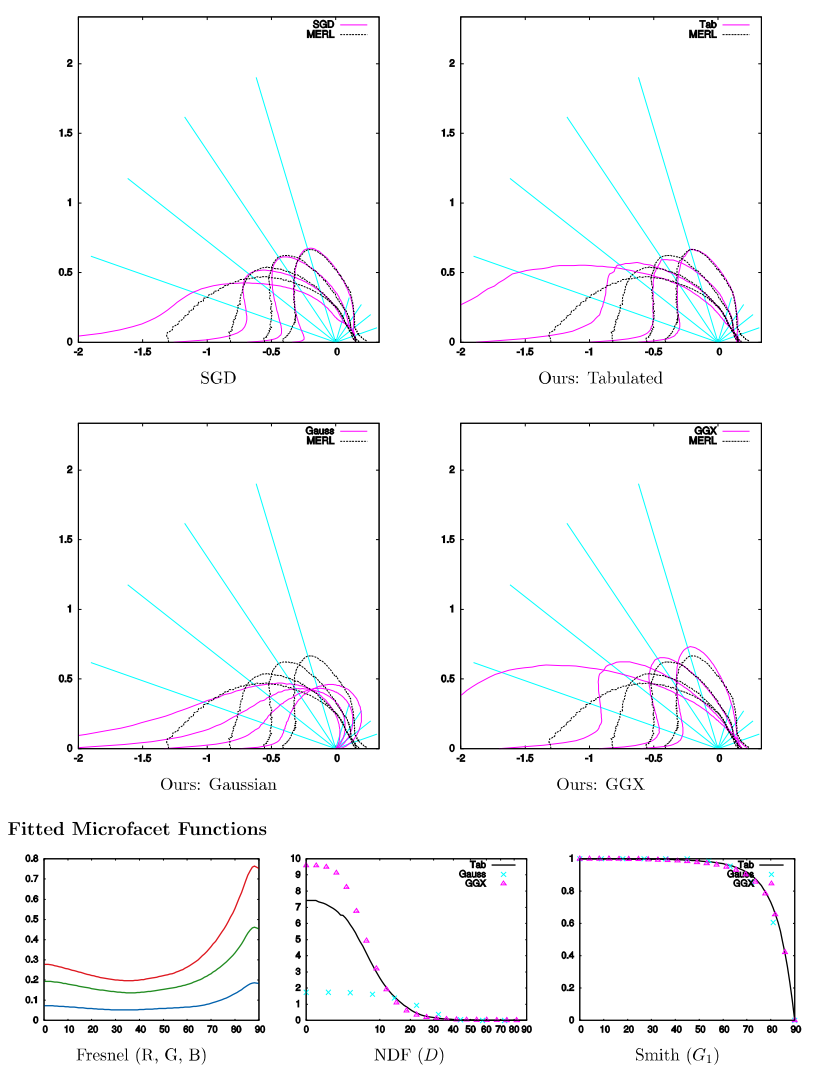


\section{Accuracy}

Mean delta-E difference image on the MERL database

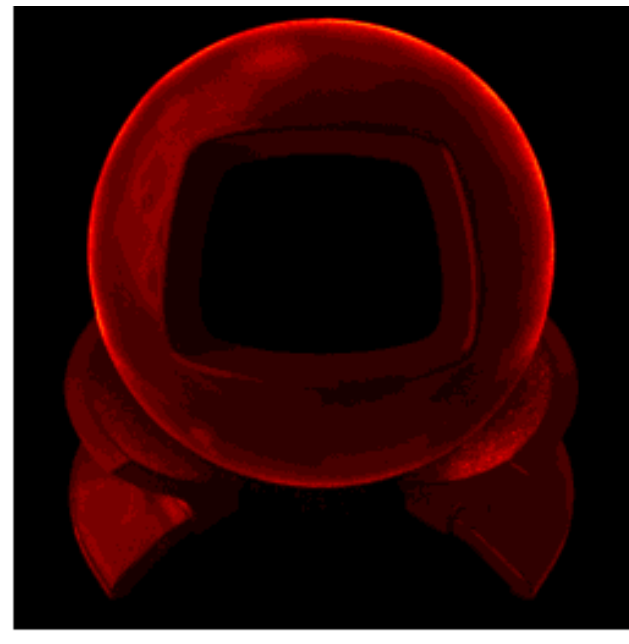

SGD [BSH12]

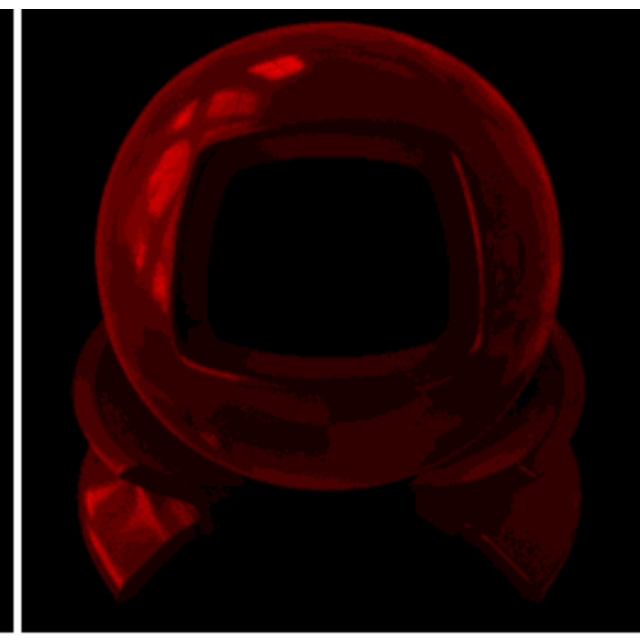

Ours: Tabulated

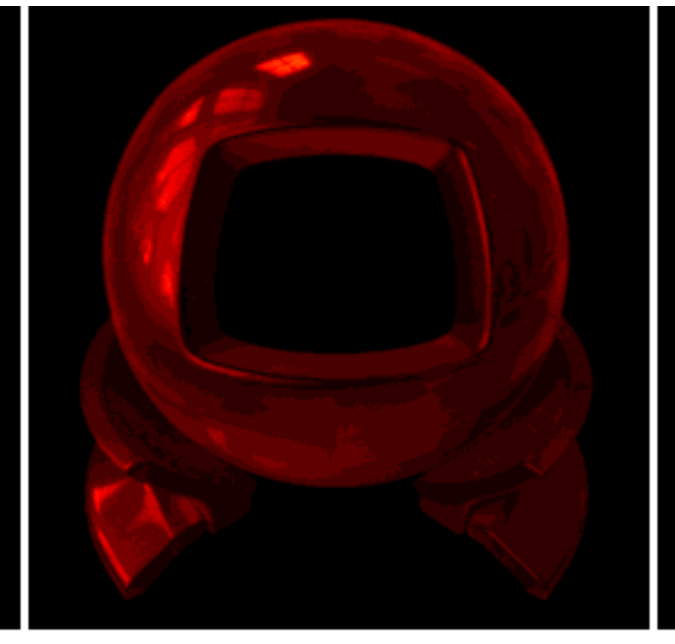

Ours: GGX

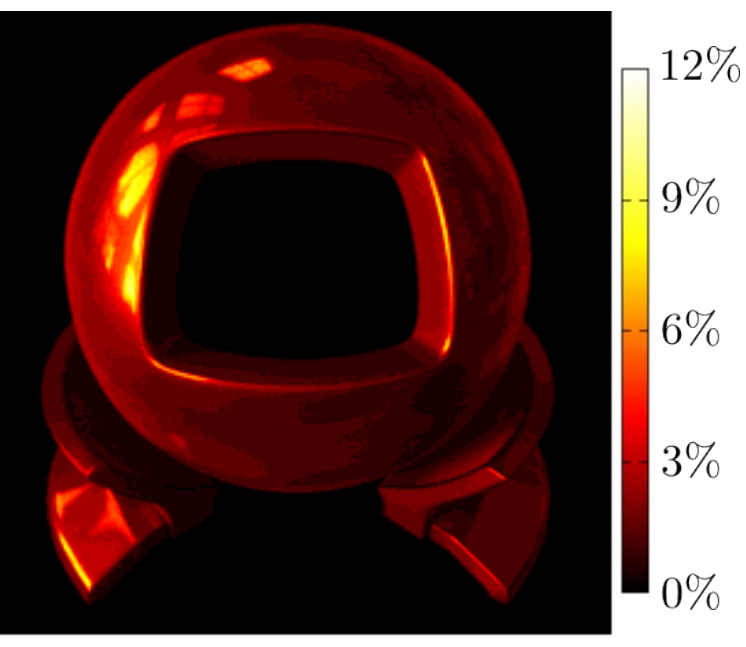

Ours: Beckmann 


\section{Contribution}

Overview

- Robust tabulation

- Robust parametric fits

Key properties

- Robustness

- Simplicity

- Speed

- Reproducibility

$$
\begin{gathered}
F_{0} D(\mathbf{o})=\int_{\Omega_{+}} K(\mathbf{o}, \mathbf{h}) D(\mathbf{h}) d \omega_{h} \\
\Rightarrow F_{0} \cdot \mathbf{d}=\mathbf{K} \cdot \mathbf{d}
\end{gathered}
$$

NDF coefficients

nonnegative matrix

\section{Perron-Frobenius theorem}

the solution is always the eigenvector with the largest magnitude 


\section{Contribution}

Novel fitting algorithm

- Robust tabulation

- Robust parametric fits

Key properties

- Robustness

- Simplicity

- Speed

- Reproducibility
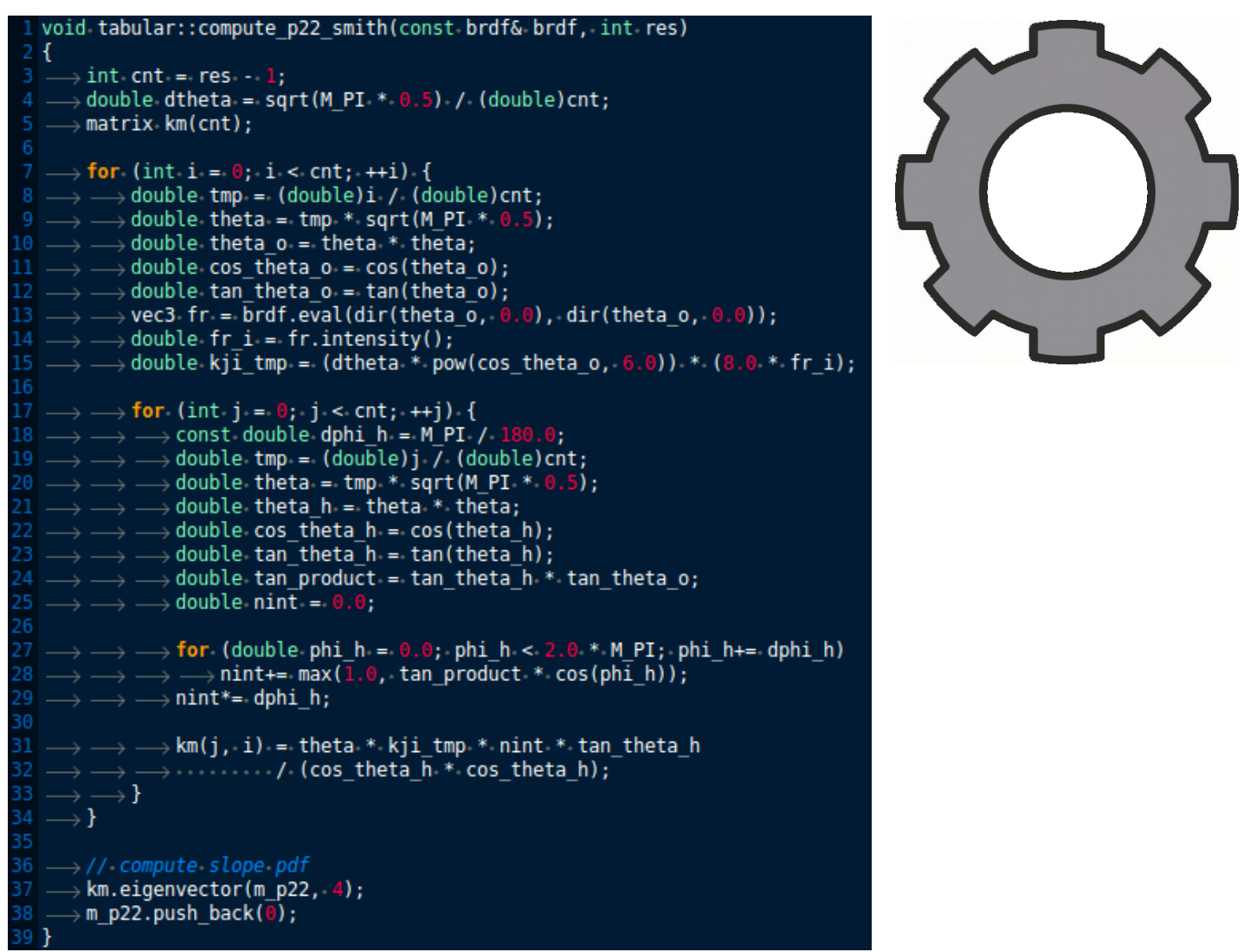


\section{Contribution}

Novel fitting algorithm

- Robust tabulation

- Robust parametric fits

Key properties

- Robustness

- Simplicity

- Speed

- Reproducibility

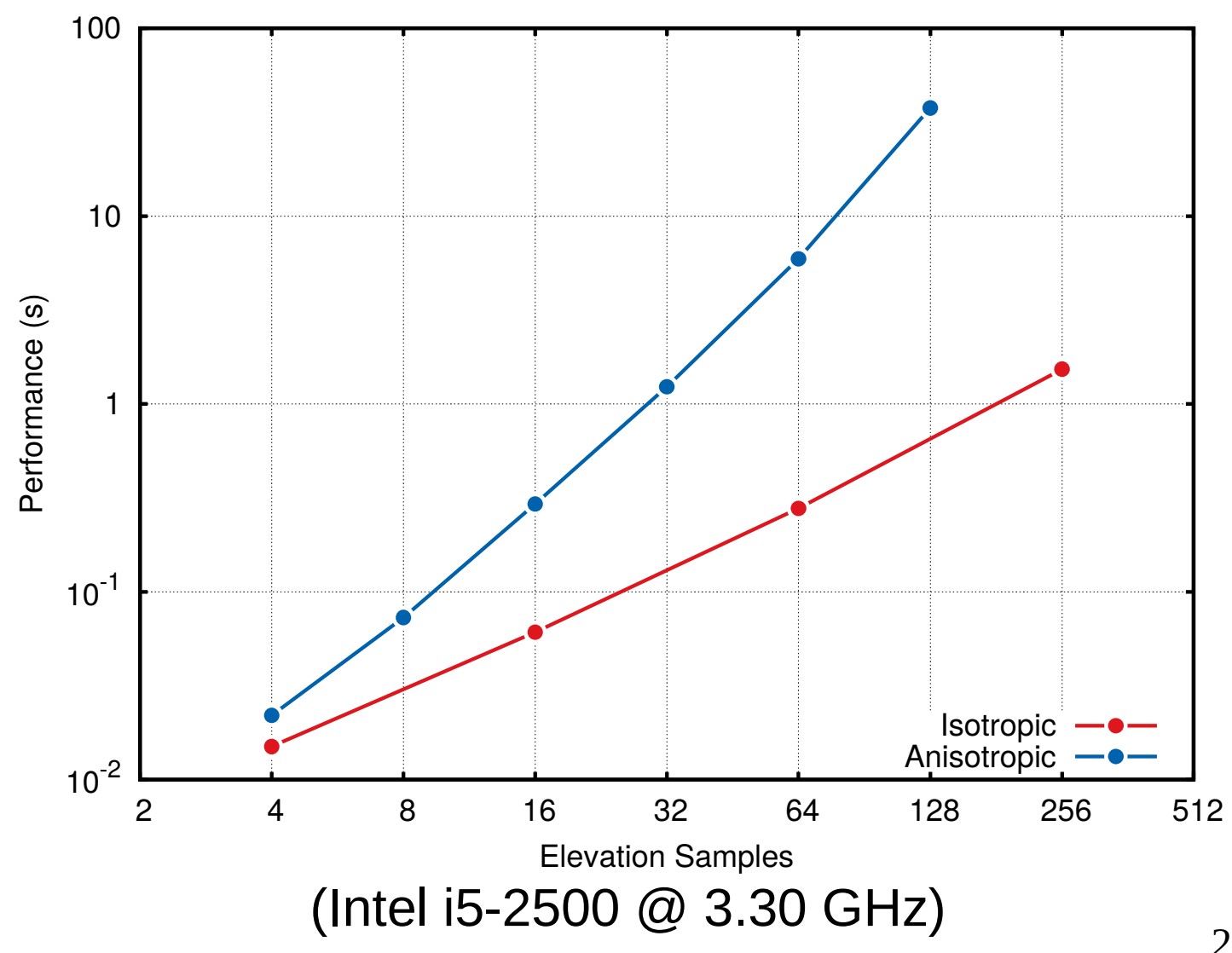




\section{Contribution}

Novel fitting algorithm

- Robust tabulation

- Robust parametric fits

Key properties

- Robustness

- Simplicity

- Speed

- Reproducibility

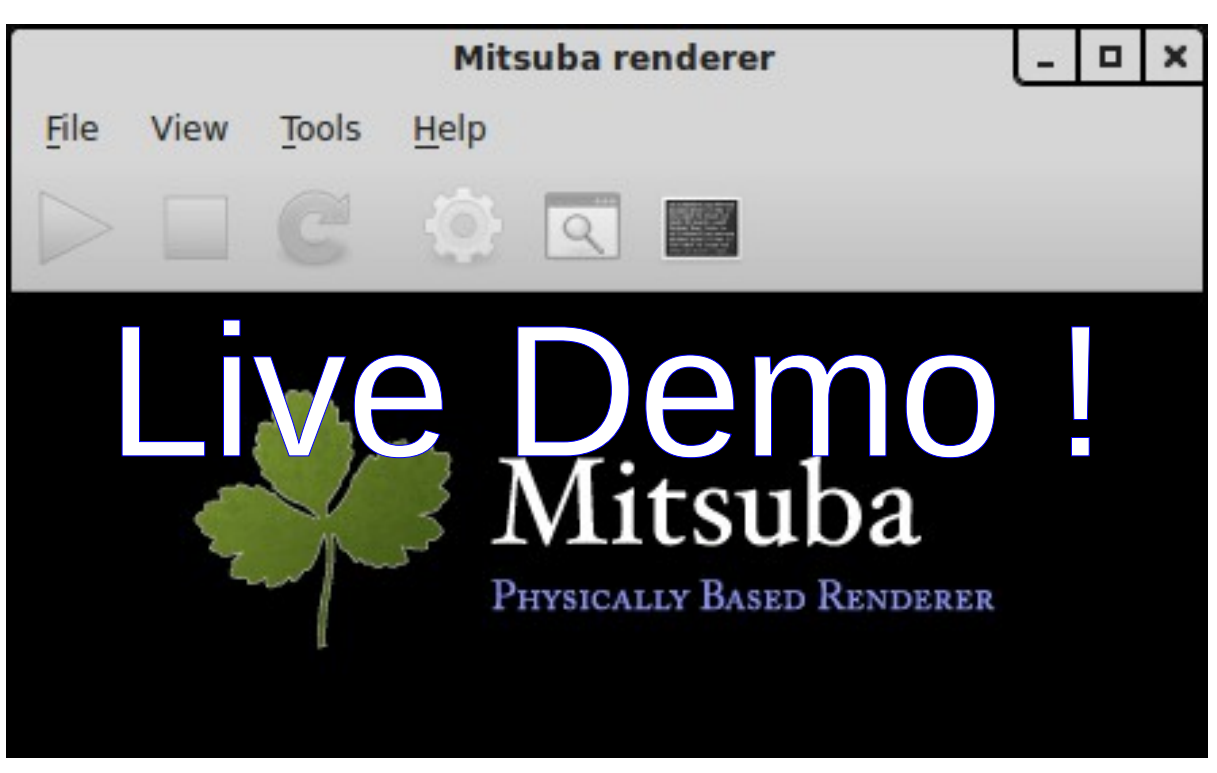

Source code:

http://github.com/jdupuy/dj_brdf 


\section{Artistic Control}

$$
D=P\left(\frac{\tilde{x}_{h}}{\alpha_{x}}, \frac{\tilde{y}_{h}}{\alpha_{y}}\right) \frac{\sec ^{4} \theta_{h}}{\alpha_{x} \alpha_{y}}
$$

Tabulate the slope PDF

- Roughness $\propto$ stretch $^{-1}$

- Efficient BRDF evaluation

- Efficient BRDF sampling

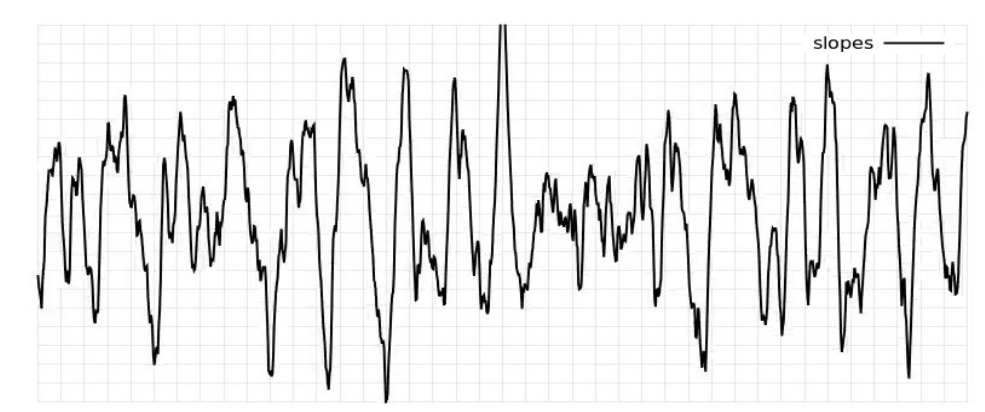




\section{Editing blue-metallic-paint}

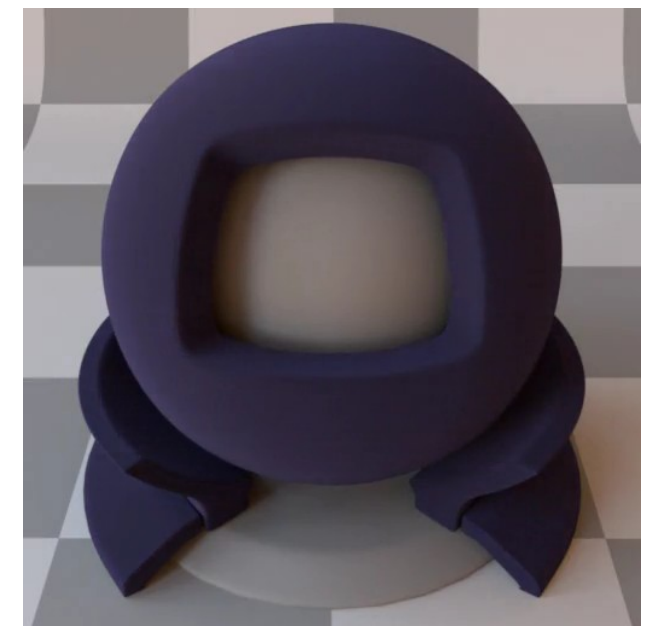

Isotropic stretch

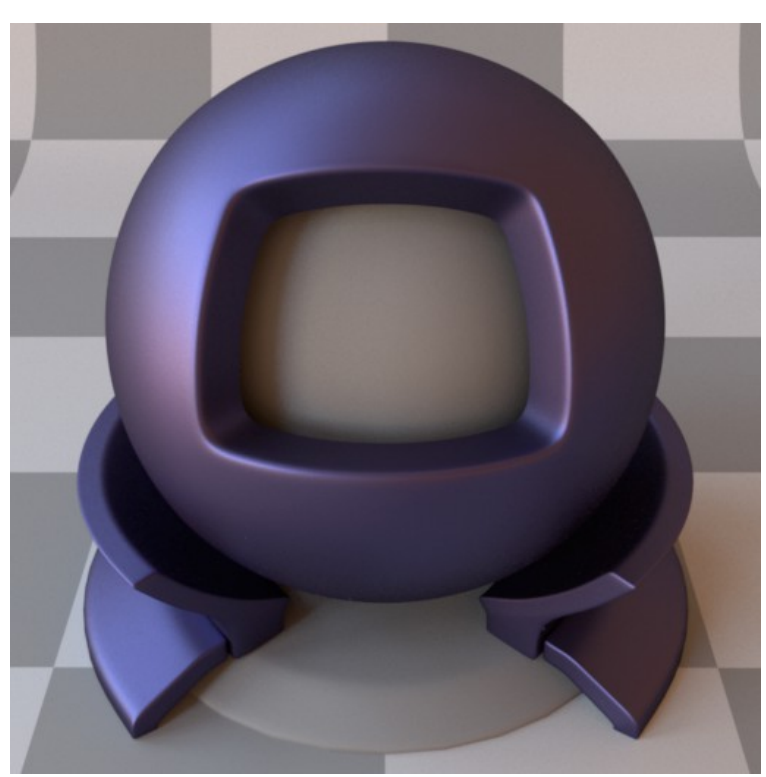

Acquired data

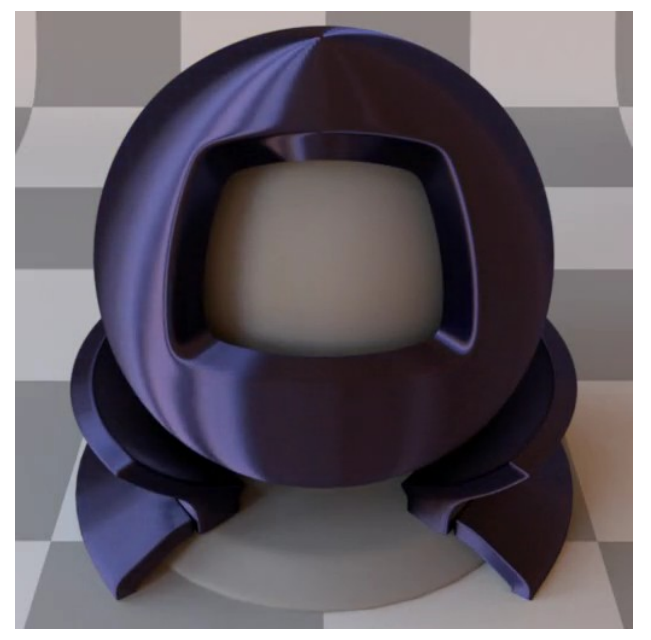

Anisotropic stretch 


\section{Roughness Textures}
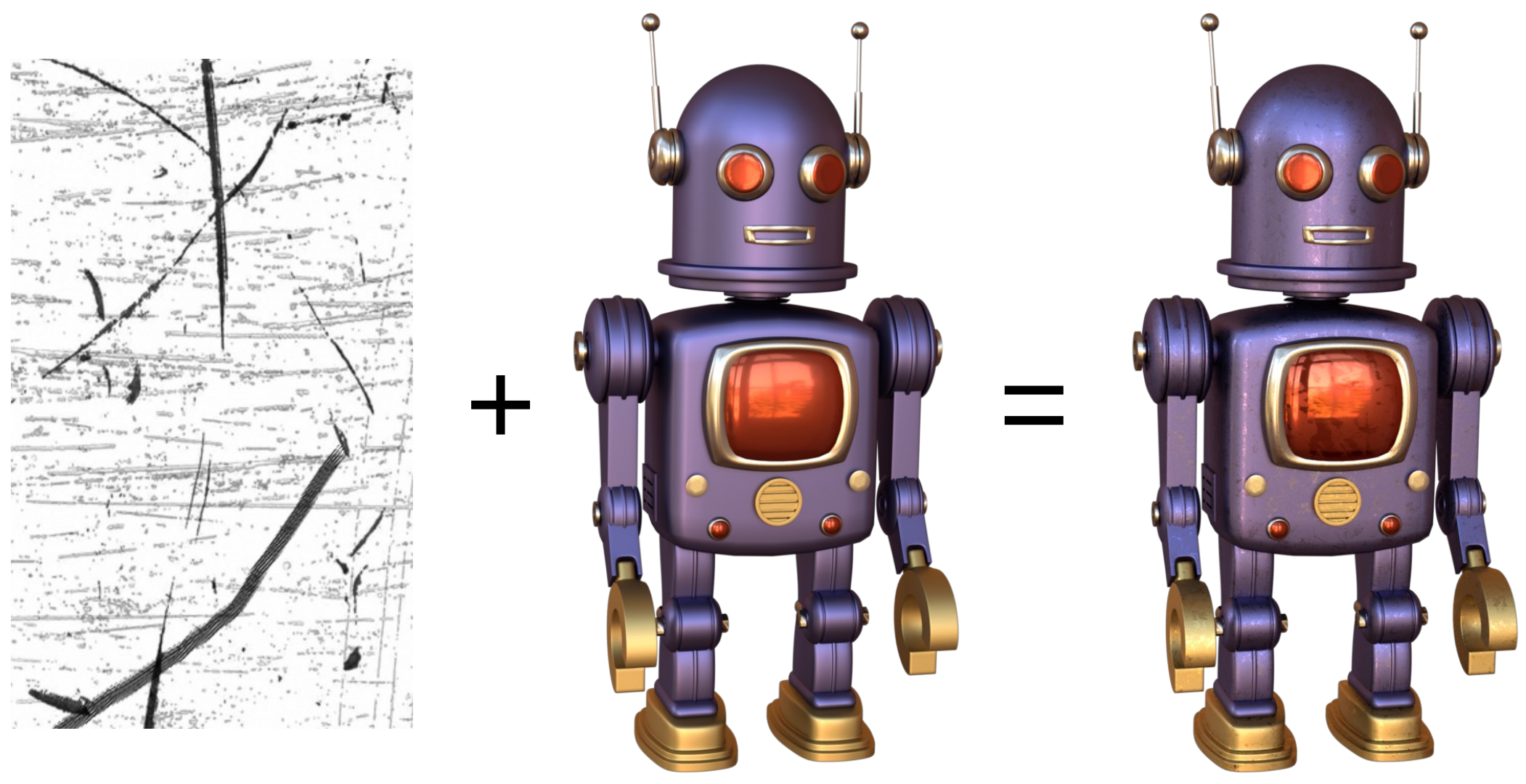

Robot model courtesy of LAGOA 


\section{Roughness Textures}
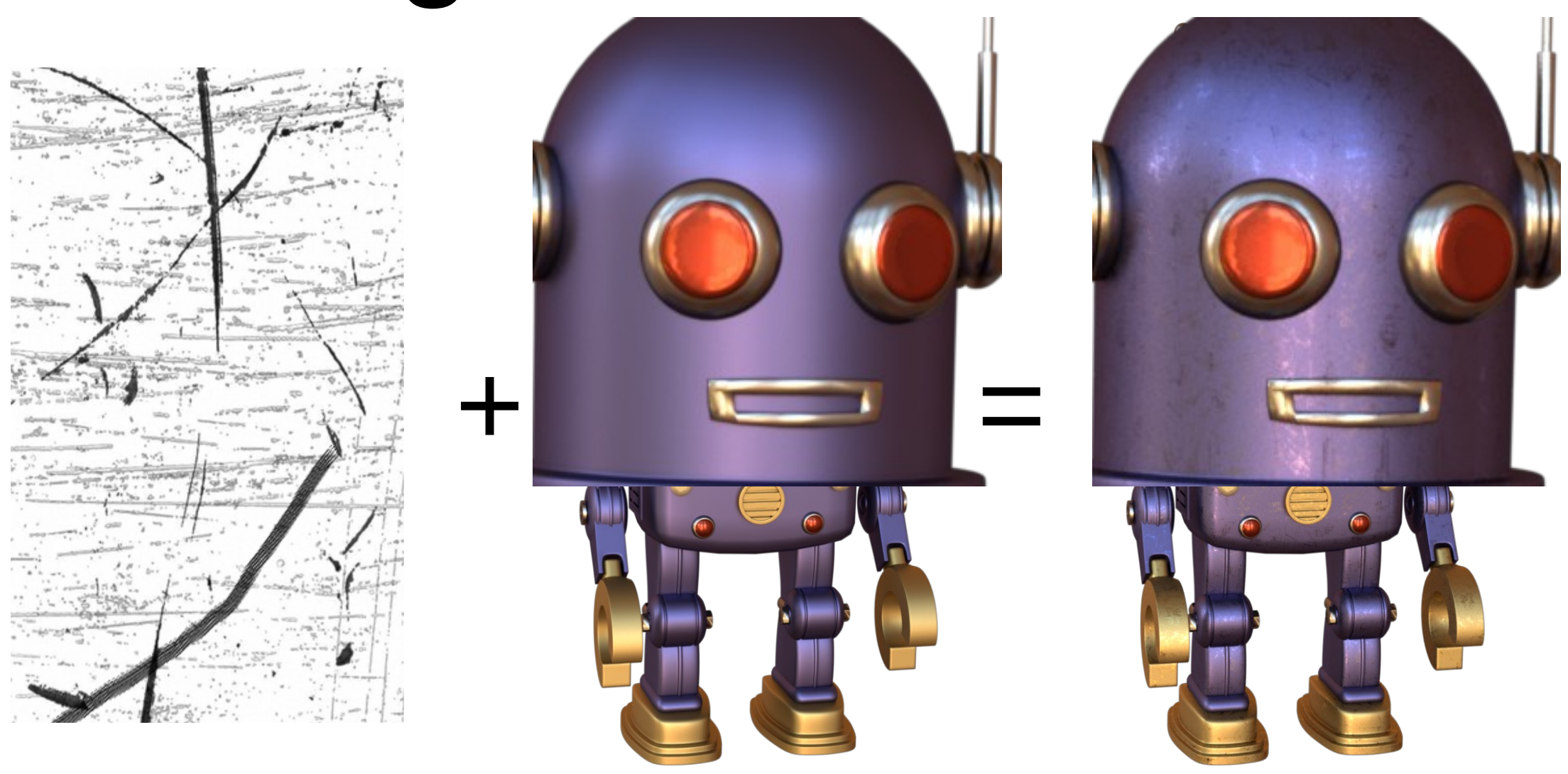

Robot model courtesy of LAGOA 


\section{Memory Consumption}

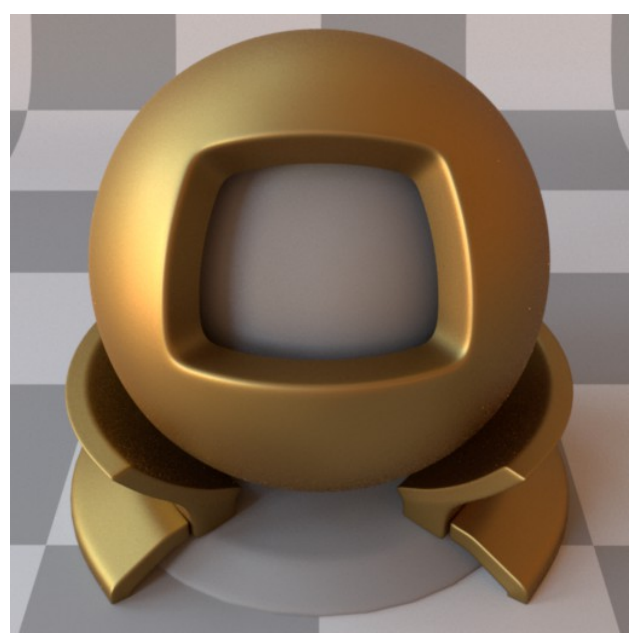

Acquired Data $35 \mathrm{MB}$

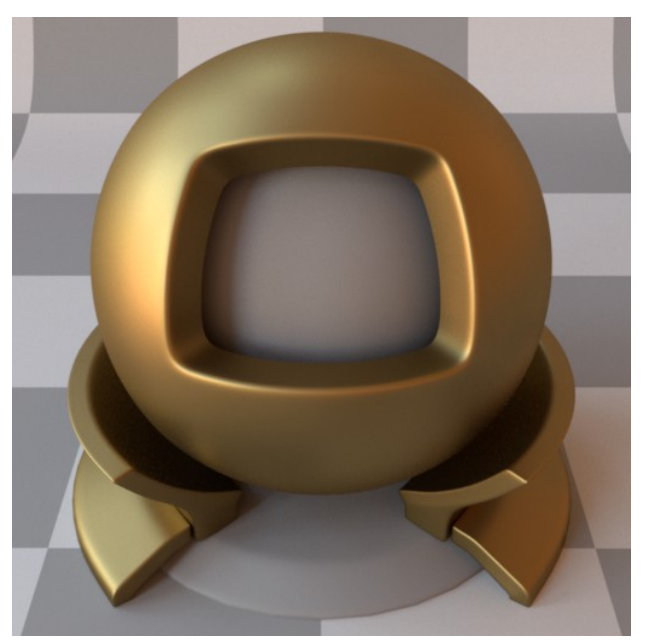

Ours: Tabulated

$2.1 \mathrm{~KB}$

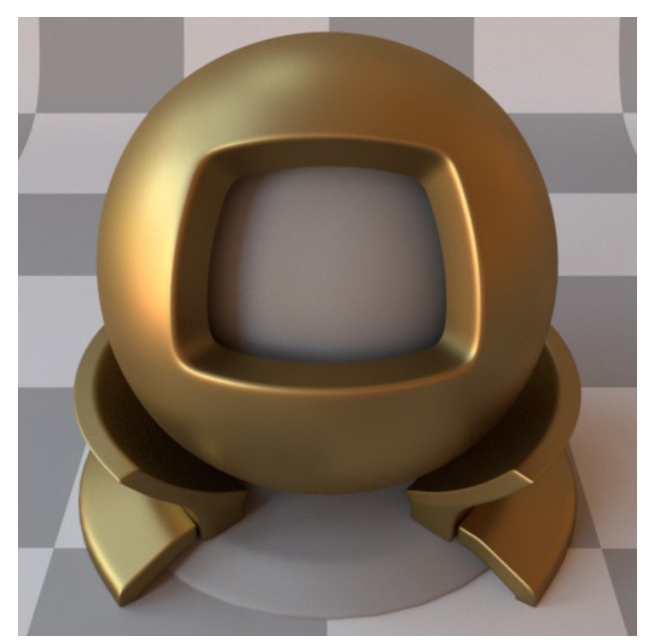

Ours: GGX $1 \mathrm{~KB}$

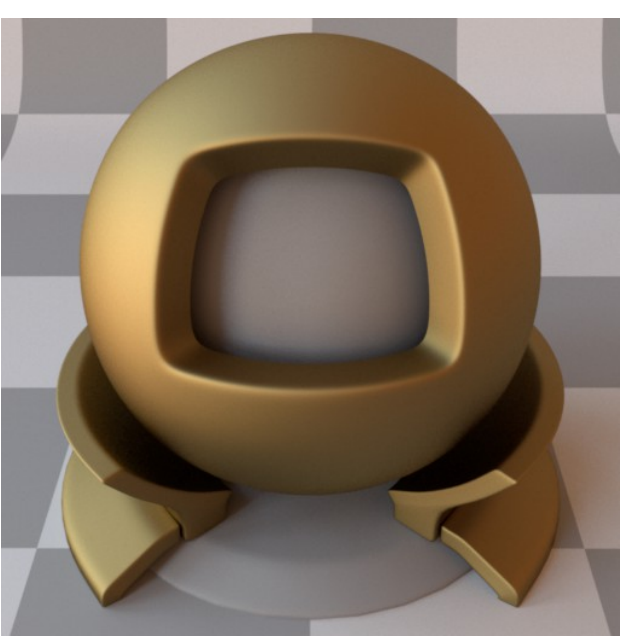

Ours: Beckmann $1 \mathrm{~KB}$ 


\section{Conclusion}

- Simple fitting technique

- Robust

- Fast

- Reproducible

- Future work

- Extend microfacet models (multiple bounces, layers, ...)

- Invert BTDF, BSSRDF, and SVBRDF

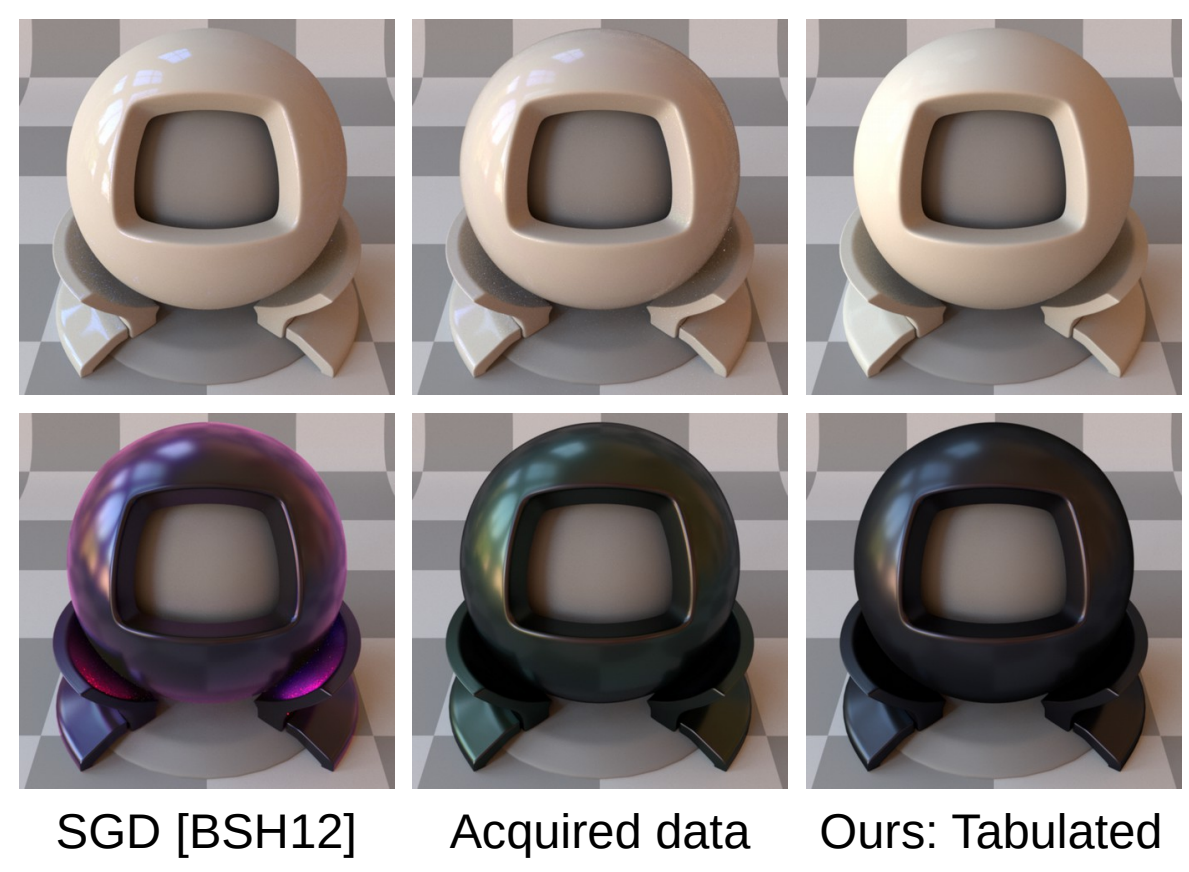




\section{Acknowledgments}

- Laurent Pujo-Menjouet and Ionel Ciuperca for their input on integral equations

- Thiago Da Costa and Arno Zinke for providing us with the LAGOA robot asset

- Brent Burley for discussing BRDF authoring in production 


\section{Questions}

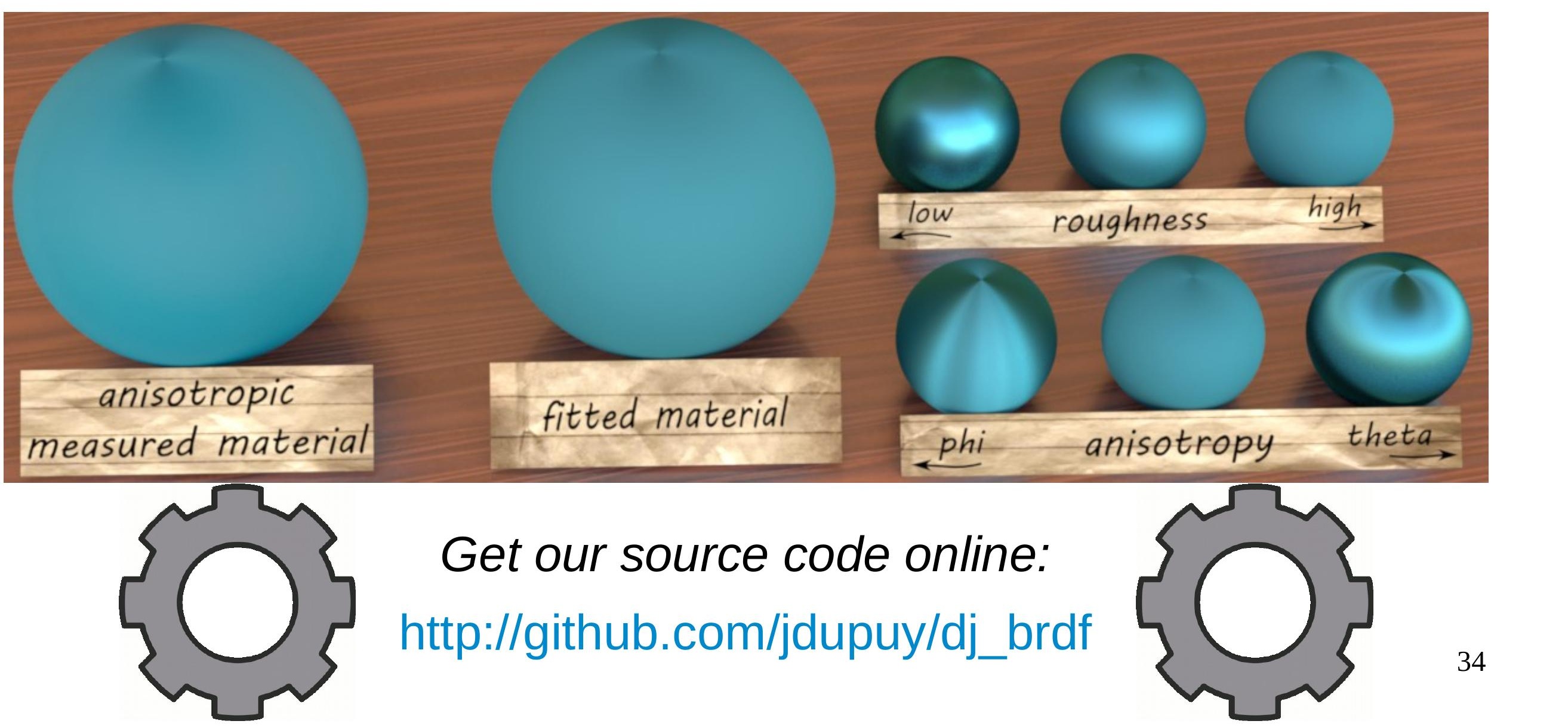




\section{Fail Case: Layers}

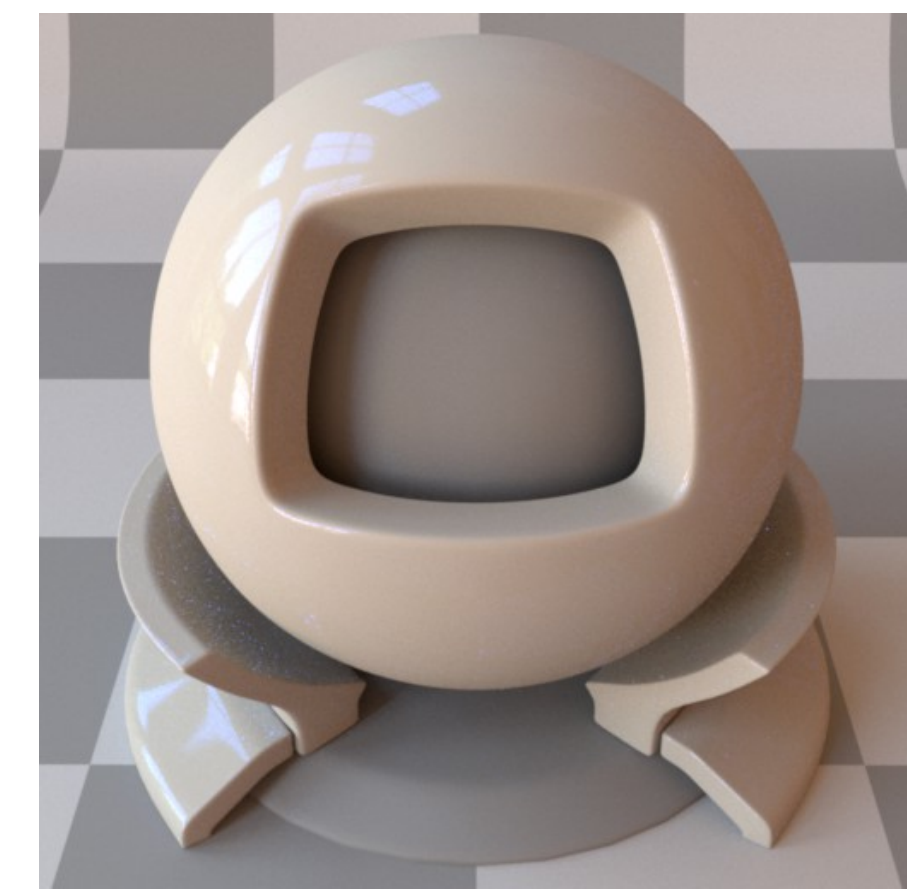

SGD [BSH12]

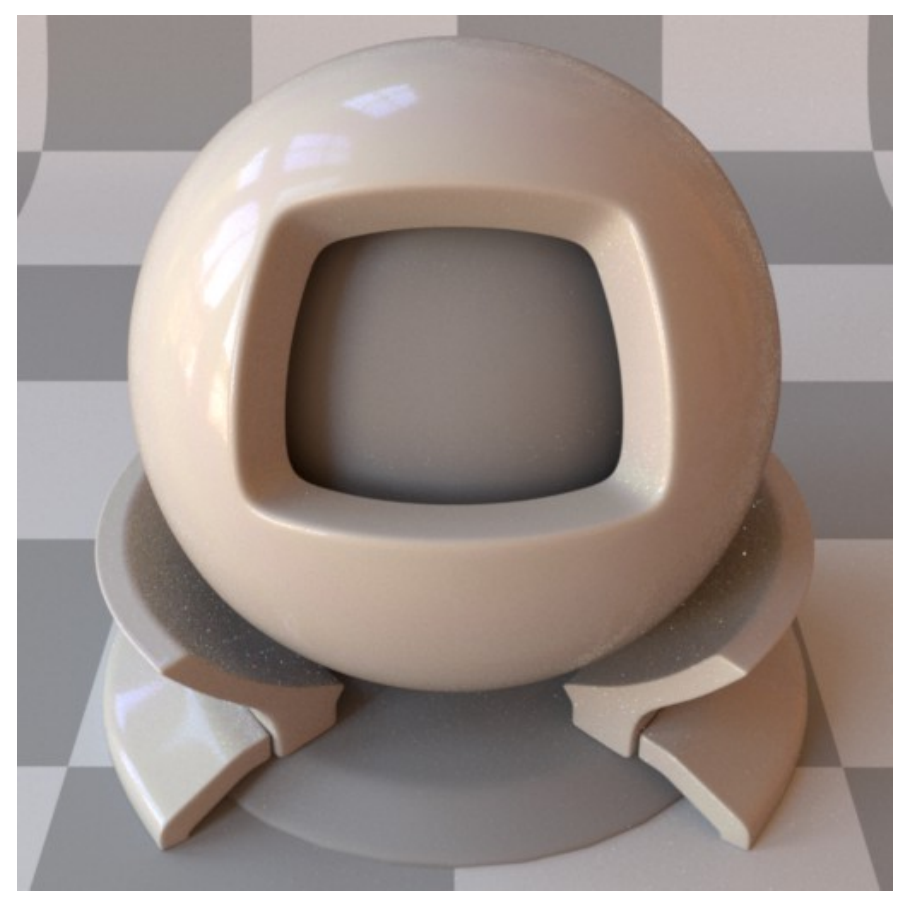

Acquired data

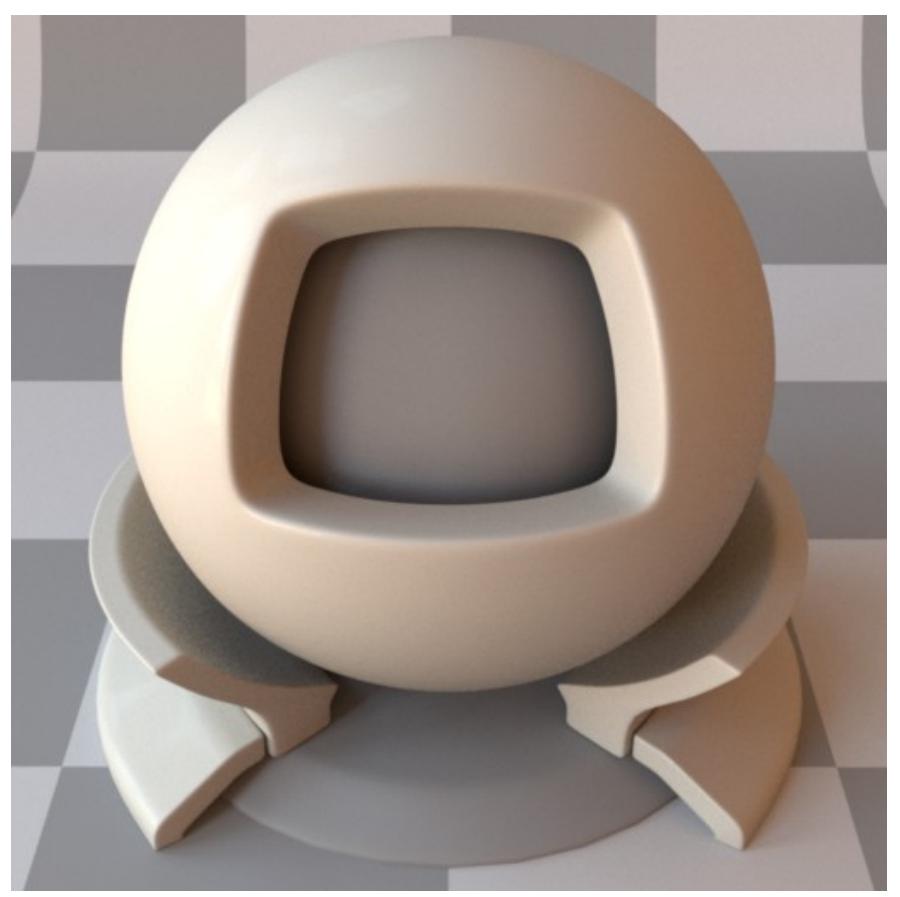

Ours: Tabulated

(MERL alumnia-oxide) 


\section{Microfacet BRDFs}

$$
f_{r}=\frac{F \cdot D \cdot G}{4 \cdot \cos \theta_{i} \cdot \cos \theta_{o}}
$$

Modular components

- Fresnel term $F$

- Distribution of normals $D$

- Roughness $\alpha$

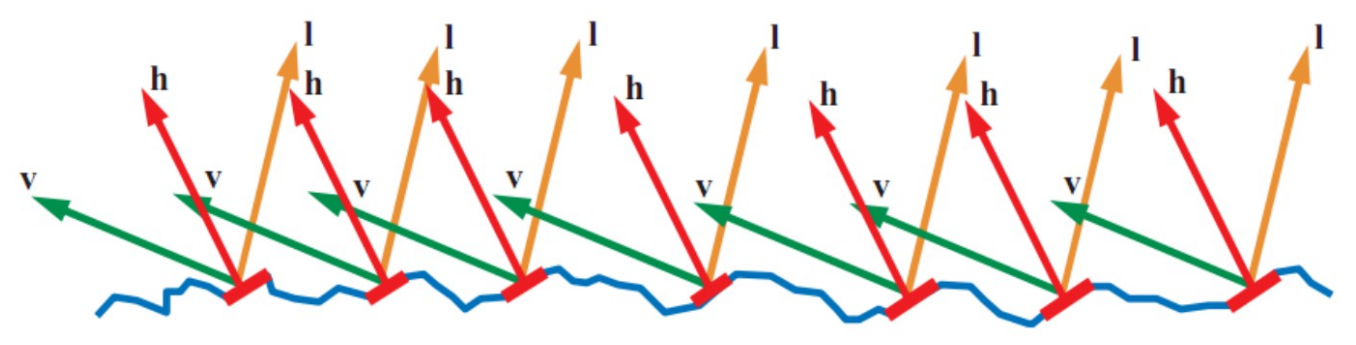

- Geometric factor $G$

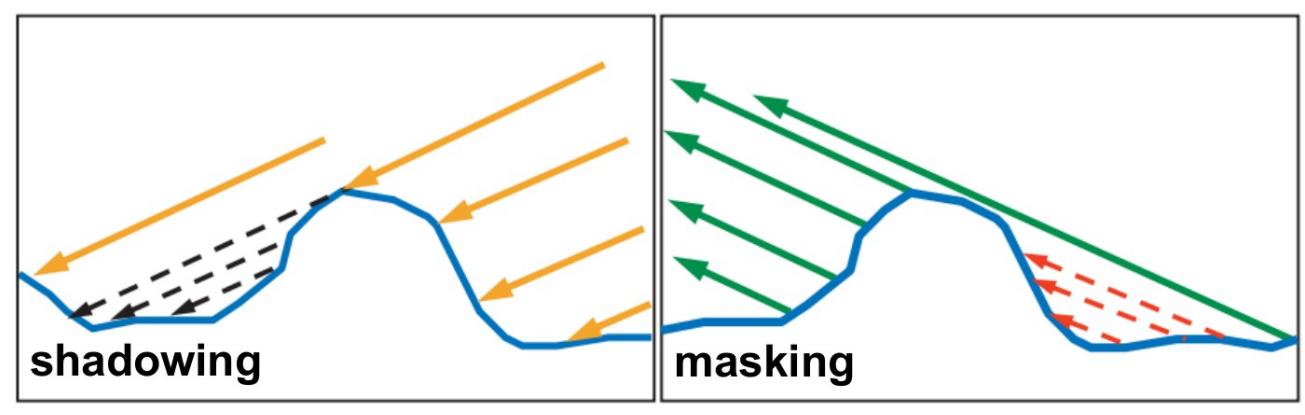




\section{Fresnel}

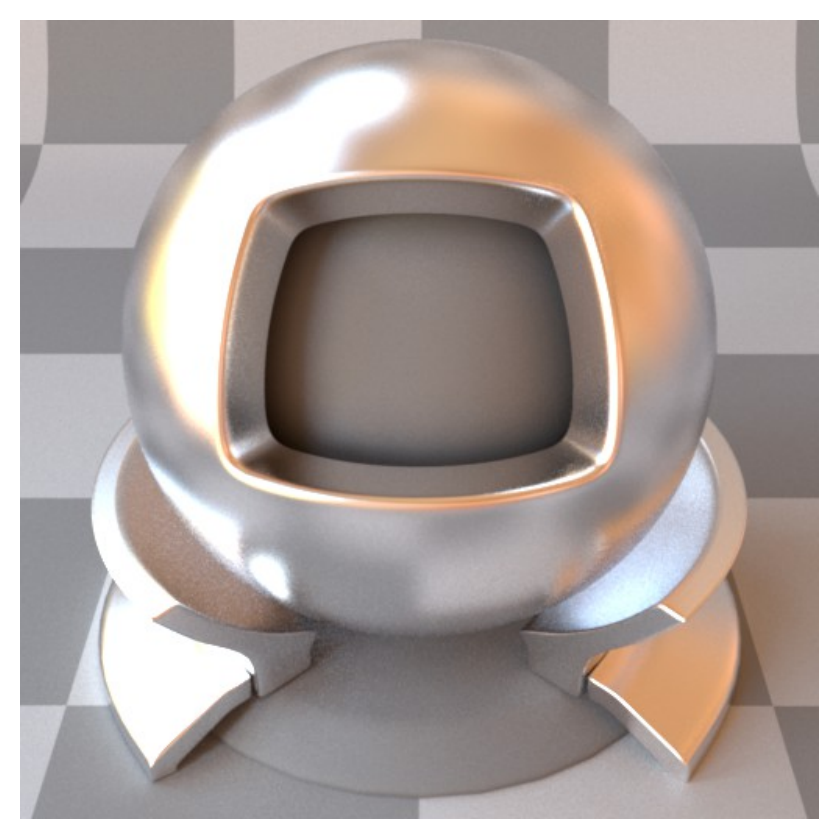

Aluminum

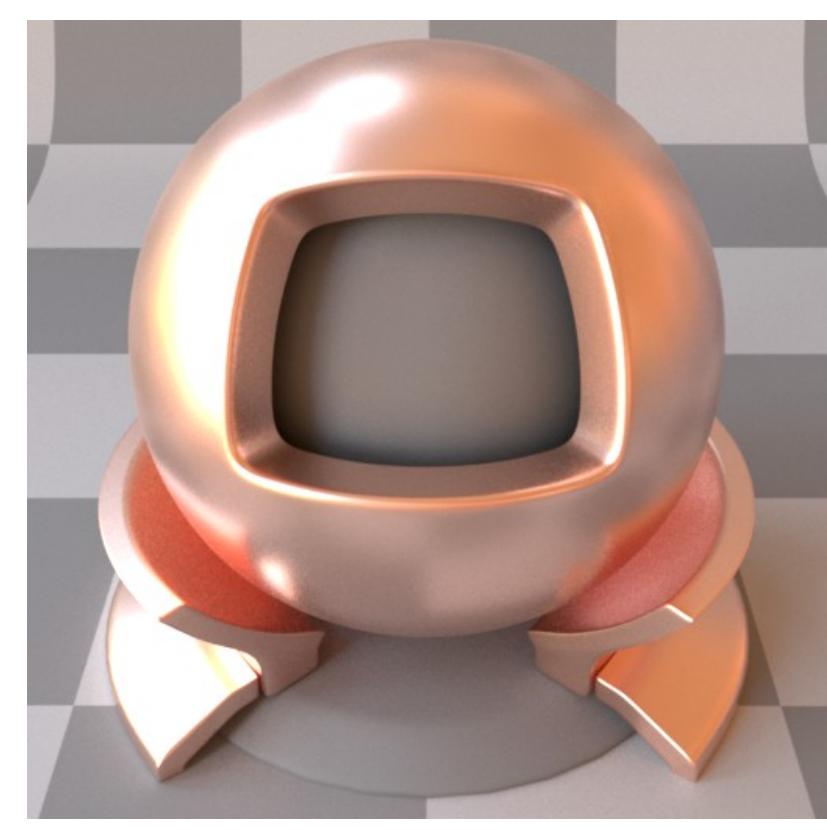

Copper

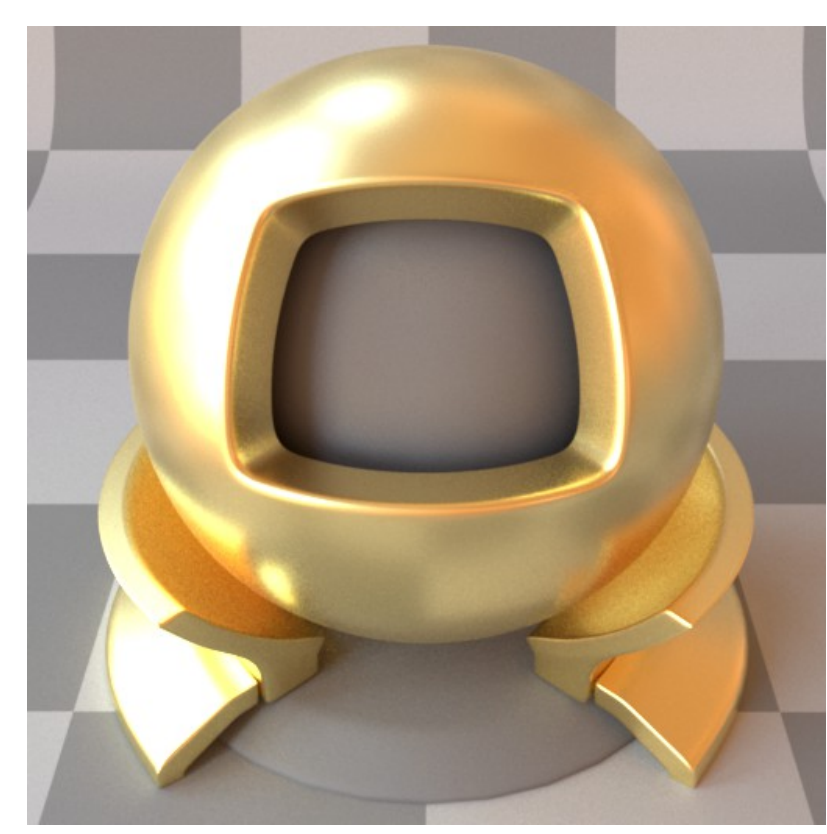

Gold 


\section{Distribution of Normals}

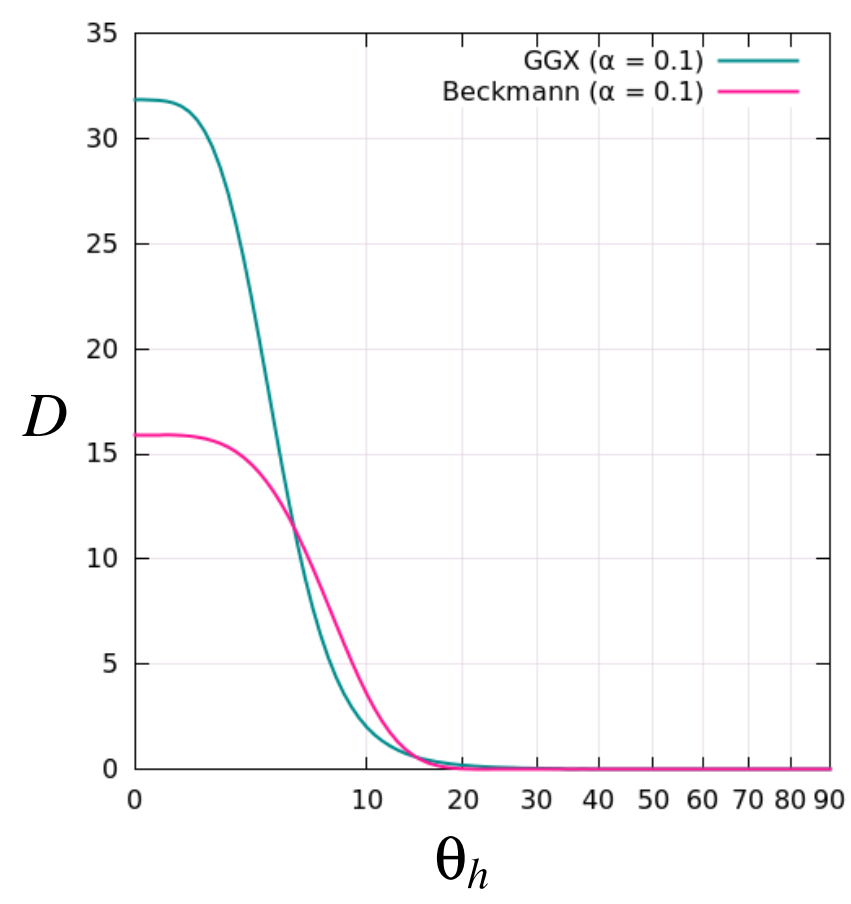

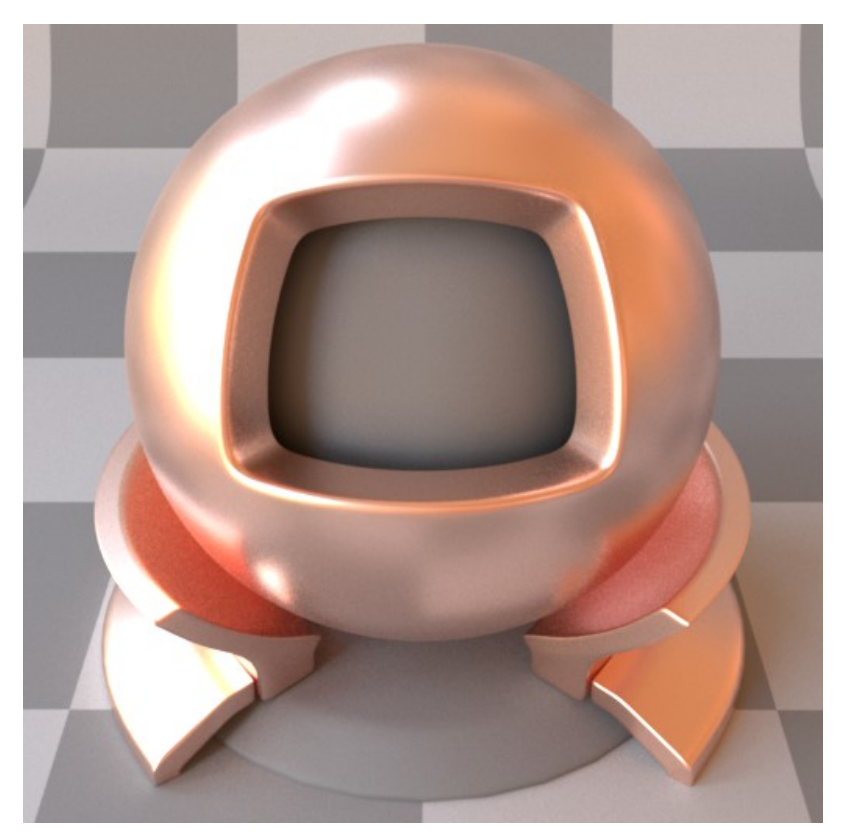

GGX

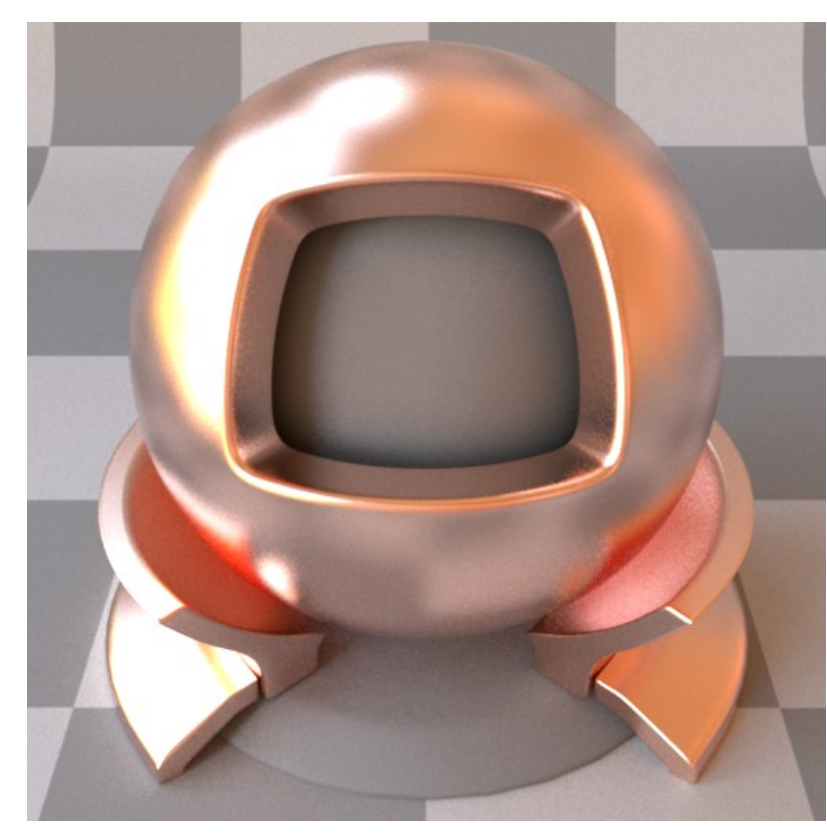

Beckmann 


\section{Roughness}
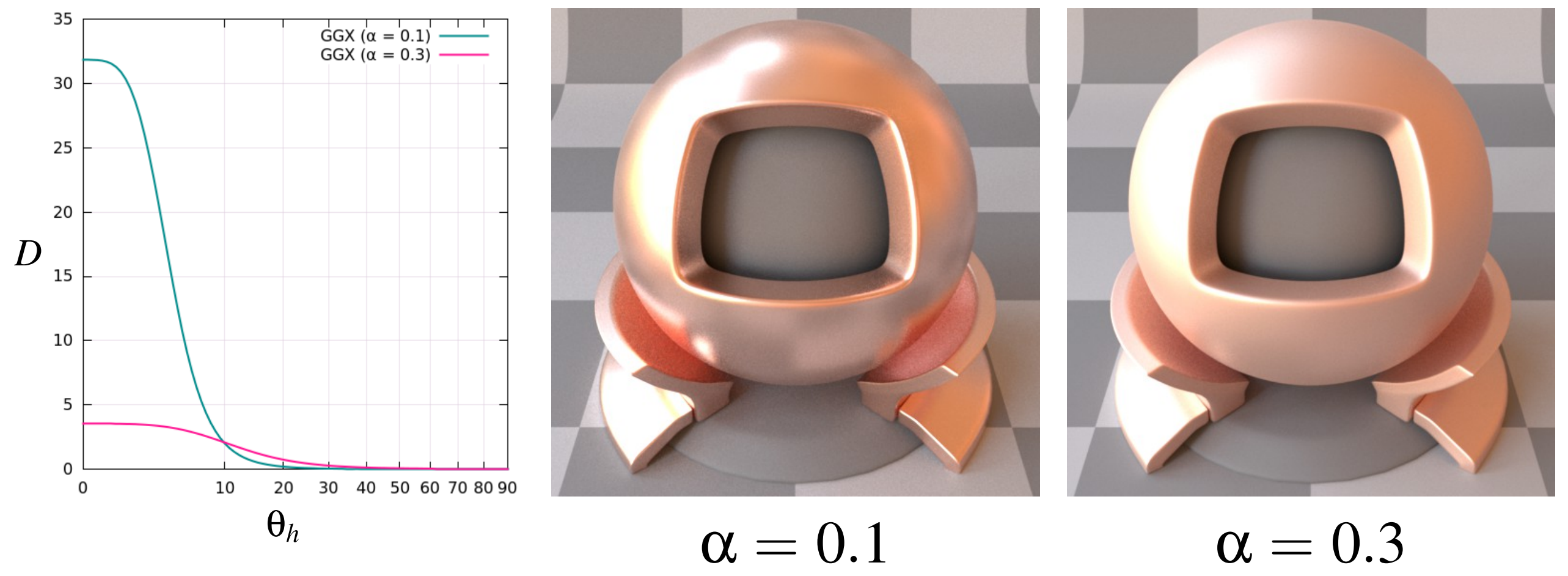


\section{Geometric Factor}

$$
\begin{gathered}
G=\min \left(G_{1}(\mathbf{h}, \mathbf{i}), G_{1}(\mathbf{h}, \mathbf{o})\right) \\
G_{1}(\mathbf{h}, \mathbf{k})=\min \left(1,2 \frac{\cos \theta_{h} \cos \theta_{k}}{\mathbf{k h}}\right) \\
G=\frac{G_{1}(\mathbf{i}) G_{1}(\mathbf{o})}{G_{1}(\mathbf{i})+G_{1}(\mathbf{o})-G_{1}(\mathbf{i}) G_{1}(\mathbf{o})} \\
G_{1}(\mathbf{k})=\frac{\cos \theta_{k}}{\int_{\Omega_{+}} \mathbf{k h} D(\mathbf{h}) d \omega_{h}}
\end{gathered}
$$

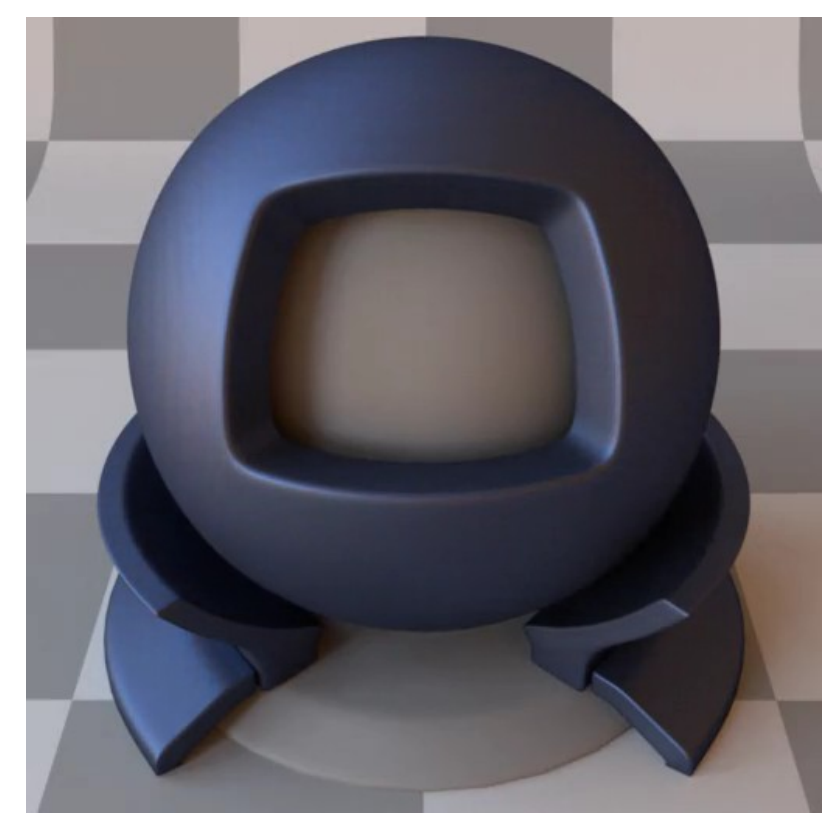

Smith

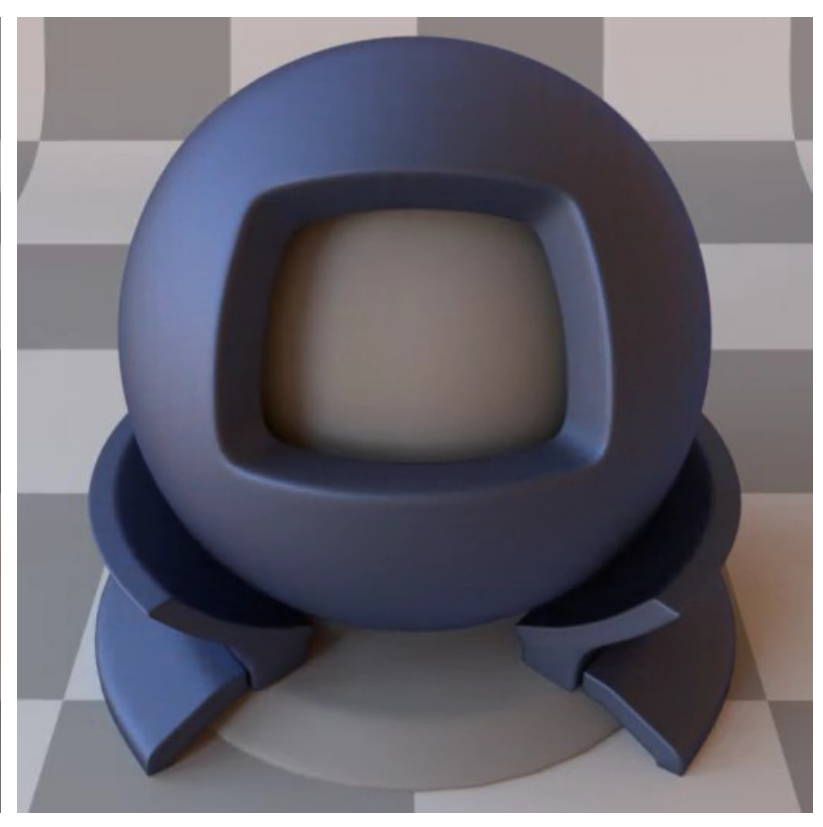

V Cavities 


\section{Inverting the BRDF}

$$
f_{r}(\mathbf{i}, \mathbf{0})=\frac{F \cdot D \cdot G}{4 \cdot \cos \theta_{i} \cdot \cos \theta_{o}}
$$

Given an input material, can we retrieve a Fresnel term, a distribution of normals, and a geometric factor ?

Too difficult :( 


\section{Inverting the Smith Model}

$$
\begin{aligned}
& f_{r}=\frac{F \cdot D \cdot G}{4 \cdot \cos \theta_{i} \cdot \cos \theta_{o}} \\
& G=\frac{G_{1}(\mathbf{i}) G_{1}(\mathbf{o})}{G_{1}(\mathbf{i})+G_{1}(\mathbf{o})-G_{1}(\mathbf{i}) G_{1}(\mathbf{o})} \\
& G_{1}(\mathbf{k})=\frac{\cos \theta_{k}}{\int_{\Omega_{+}} \mathbf{k h} D(\mathbf{h}) d \omega_{h}}
\end{aligned}
$$
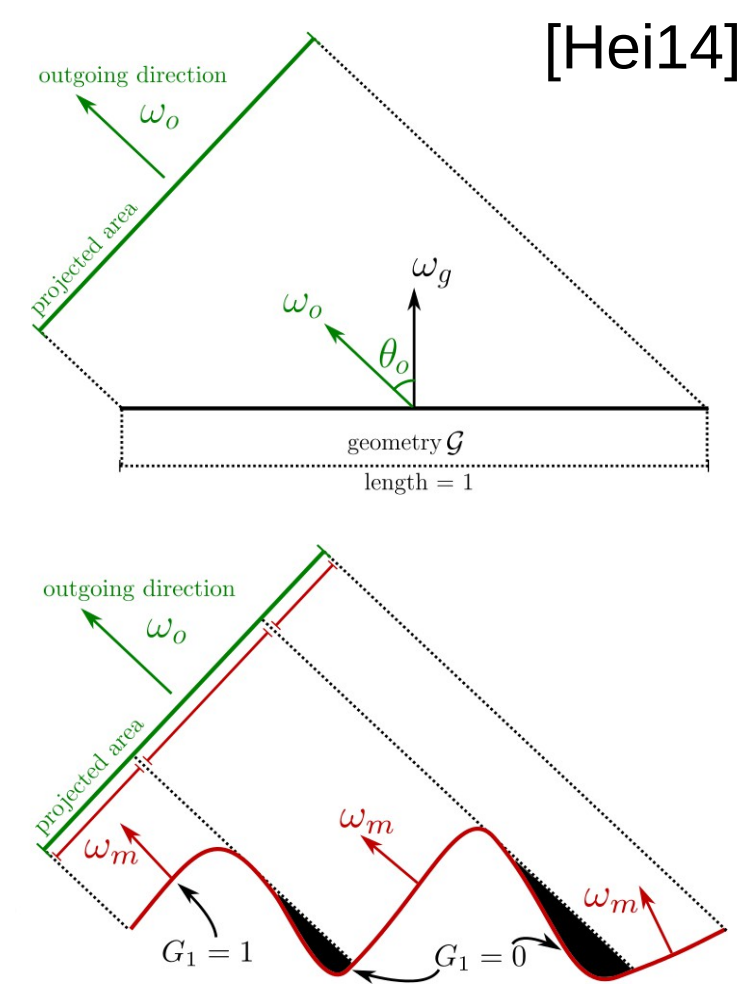

Given an input material, can we retrieve a Fresnel term and a distribution of normals? 